\title{
Star formation in the Trifid Nebula ${ }^{\star}$ Cores and filaments
}

\author{
B. Lefloch ${ }^{1}$, J. Cernicharo ${ }^{2,1}$, and J. R. Pardo ${ }^{2}$ \\ ${ }^{1}$ Laboratoire d'Astrophysique de l'Observatoire de Grenoble, 414 rue de la Piscine, 38041 Grenoble Cedex, France \\ e-mail: Bertrand.lefloch@obs.ujf-grenoble.fr \\ 2 DAMIR, CSIC, C./Serrano 121, 28006 Madrid, Spain
}

Received 28 April 2008 / Accepted 30 June 2008

\section{ABSTRACT}

\begin{abstract}
Aims. We aim to characterize the properties of the prestellar and protostellar condensations to understand the star formation processes at work in a young HII region

Methods. We have obtained maps of the $1.25 \mathrm{~mm}$ thermal dust emission and the molecular gas emission over a region of $20^{\prime} \times 10^{\prime}$ around the Trifid Nebula (M20), with the IRAM $30 \mathrm{~m}$ and the CSO telescopes as well as in the mid-infrared wavelength with ISO and SPITZER. Our survey is sensitive to features down to $N\left(\mathrm{H}_{2}\right) \sim 10^{22} \mathrm{~cm}^{-2}$ in column density.

Results. The cloud material is distributed in fragmented dense gas filaments $\left(n\left(\mathrm{H}_{2}\right)\right.$ of a few $\left.10^{3} \mathrm{~cm}^{-3}\right)$ with sizes ranging from 1 to 10 pc. A massive filament, WF, with properties typical of Infra Red Dark Clouds, connects M 20 to the W28 supernova remnant. We find that these filaments pre-exist the formation of the Trifid and were originally self-gravitating. The fragments produced are very massive (typically $100 M_{\odot}$ or more) and are the progenitors of the cometary globules observed at the border of the HII region. We could identify 33 cores, 16 of which are currently forming stars. Most of the starless cores have typical $\mathrm{H}_{2}$ densities of a few $10^{4} \mathrm{~cm}^{-3}$. They are usually gravitationally unbound and have low masses of a few $M_{\odot}$. The densest starless cores $\left(\right.$ several $10^{5} \mathrm{~cm}^{-3}$ ) are located in condensation $\mathrm{TC} 0$, currently hit by the ionization front, and may be the site for the next generation of stars. The physical gas and dust properties of the cometary globules have been studied in detail and have been found very similar. They all are forming stars. Several intermediate-mass protostars have been detected in the cometary globules and in the deeply embedded cores. Evidence of clustering has been found in the shocked massive cores TC3-TC4-TC5.

Conclusions. M 20 is a good example of massive-star forming region in a turbulent, filamentary molecular cloud. Photoionization appears to play a minor role in the formation of the cores. The observed fragmentation is well explained by MHD-driven instabilities and is usually not related to M 20. We propose that the nearby supernova remnant W28 could have triggered the formation of protostellar clusters in nearby dense cores of the Trifid.
\end{abstract}

Key words. stars: formation - ISM: clouds - HII regions - Herbig-Haro objects - dust, extinction

\section{Introduction}

Many observations suggest that HII regions could play an important role in spreading star formation throughout the Galaxy. As reviewed in Elmegreen (2002), most of the embedded young stellar clusters in the solar neighbourhood are adjacent to HII regions excited by more evolved stars: Orion, Perseus OB2, the Rosette nebula, W3-5, M 17, to name but a few. Yamaguchi et al. (1999) estimate that $10 \%-30 \%$ of stars in mass in the Galaxy are formed under the influence of adjacent HII regions. In particular, the condensations encountered at the border of the HII regions, the so-called bright-rimmed Globules and cometary globules (CGs), have long been identified as stellar nurseries or "incubators" (Sugitani et al. 1991, 1994; Lefloch et al. 1997; Dobashi et al. 2001). These condensations are local clumps which emerge from the expanding molecular layer surrounding the nebula. Several theoretical and observational studies have shown the important role played by the Ly-continuum radiation of the nebula in the evolution of these condensations, which is now well understood (Bertoldi 1989; Lefloch \& Lazareff 1994, 1995). Systematic studies show that these "stellar factories" tend

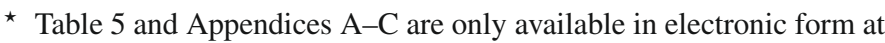
http://www . aanda.org to form more massive and luminous (proto)stars, with luminosities $\geq 10^{3} L_{\odot}$ (Sugitani et al. 1991; Dobashi 2001). It is estimated that more than $15 \%$ of the massive stars form in CGs. The question of whether the mechanical and radiative luminosities of OB stars can trigger the formation of subsequent generations of stars, in particular of massive stars, in these condensations is still an open question.

The "collect and collapse" model, first proposed by Elmegreen \& Lada (1977), is particularly interesting as it enables the formation of massive condensations and subsequently of massive objects out of an initially uniform medium. Systematic studies have shown that this mechanism was at work in some isolated Galactic HII regions and most likely responsible for the massive YSOs observed at the border of the nebulae (Deharveng et al. 2003, 2006; Zavagno et al. 2007). In the case of a turbulent environment, the molecular gas is no longer homogeneous but distributed in clumpy and filamentary structures; it is not clear however what are the most efficient processes that account for the formation of the subsequent generations of stars. Several groups have investigated the impact of HII regions on the second-generation star formation, and proposed two main mechanisms of induced star formation, either direct triggering from HII regions and other pressure mechanisms associated with hot 
stars, or gravity-driven streaming of gas along the filaments to build dense cores (Nakamura et al. 1993; Tomisaka 1995; Fiege \& Pudritz 2000; Fukuda \& Hanawa 2000).

We have started a comprehensive study of a young HII region, the Trifid Nebula (M20), small enough to allow a complete census of the young stellar and protostellar population, to address this question. The Trifid Nebula appears as a small dusty nebula in a young evolutionary age, at an heliocentric distance of $1.68 \mathrm{kpc}$ (Lynds et al. 1985). Mapping of the thermal dust and molecular gas emission of the nebula at millimeter wavelengths revealed the presence of several massive protostellar cores (dubbed TC1 to TC4), some of which are exposed to the ionizing radiation of the exciting star (Cernicharo et al. 1998, CL98). ISOCAM observations confirmed the extreme youth of the protostellar sources detected in the cores TC3 and TC4 (see also Lefloch \& Cernicharo 2000, hereafter LC00). So far, only the cometary globule TC2 has been analyzed in detail from its line and continuum emission at mid-infrared, millimeter and centimeter wavelengths (Lefloch et al. 2002, herafter L02); its protostellar content could not be characterized from the ISOCAM data, however.

The last phases of protostellar evolution in the nebula have been identified thanks to ISOCAM and SPITZER. Deep images obtained by SPITZER in the range 3.5-24 $\mu \mathrm{m}$ with IRAC and MIPS have revealed the population of low-mass objects which formed together with the exciting star of the nebula (Rho et al. 2006). In addition to 32 protostellar candidates, about 120 young stellar objects (Class II) have been detected, which are surrounded by warm dust, presumably distributed in protoplanetary disks. Analyzing the mid-infrared and centimeter emission of the central stellar cluster members, we found that their disks (components B, C, D) undergo copious photoionization (Lefloch et al. 2001, hereafter L01; Yusef-Zadeh et al. 2005).

In this article, we report on a large-scale survey of the cold dust emission around the Trifid Nebula, complemented with molecular line emission data. Our systematic mapping has allowed us to determine the evolutionary status of the SPITZER Class 0/I candidates; it reveals the presence of stellar incubators in the dense molecular material surrounding the HII region, and the progenitors of the protostellar cores. This allows us to draw a full evolutionary sequence for the incubators in the Trifid Nebula, from the early stage when they are embedded in low-density material of the parental cloud, as illustrated by the massive pre-Orion cores TC3-TC4, to the late stage when the parental cocoon is fully dissipated, leaving the protoplanetary disks exposed to the ionizing radiation of the $\mathrm{O}$ star, as observed by L01 and Yusef-Zadeh et al. (2005).

The article is organized as follows: after presenting the observational data (Sect. 2), we summarize the main results of the continuum survey (Sect. 3). We then present in Sects. 4-6 detailed studies of typical cores which have formed in the western filament (TC0), the eastern lane (TC1) and in the South of the nebula (TC2 and TC9). We discuss in Sect. 7 the implications on the star formation history of the Trifid. The main conclusions are summarized in Sect. 8.

\section{The observations}

\subsection{Continuum observations}

The ISO observations of the nebula are fully described in L02. Spitzer observations are presented and discussed in Rho et al. (2006). In all maps presented here the coordinates are given in arcsec offsets with respect to the position of HD 164429A, the
O star exciting the nebula: $\alpha=18^{\mathrm{h}} 02^{\mathrm{m}} 23.55^{\mathrm{s}}, \delta=-23^{\circ} 01^{\prime} 51^{\prime \prime}$ (J2000.0).

Observations of the $1.25 \mathrm{~mm}$ continuum emission in the Trifid were carried out in March 1996 and March 1997 at the IRAM 30 m-telescope (Pico Veleta, Spain) using the MPIfR 19-channel bolometer array. The passband of the bolometer has an equivalent bandwidth $\simeq 70 \mathrm{GHz}$ and is centered at $240 \mathrm{GHz}$. The beam size of the telescope is $11^{\prime \prime}$. The final map was obtained by combining several individual fields centered on the brightest condensations of the nebula. Each field was scanned in the horizontal direction by moving the telescope at a speed of $4^{\prime \prime}$ per sec; subsequent scans are displaced by $4^{\prime \prime}$. We used a chopping secondary at $2 \mathrm{~Hz}$ with a throw of 30 to $90^{\prime \prime}$ depending on the structure of the region to be mapped. The dual-beam maps were reduced with the IRAM software for bolometerarray data (Broguiere et al. 1995), which uses the EKH restoration algorithm (Emerson et al. 1979). Zero-order baselines were subtracted from the data prior to restoration. A few maps suffered from strong skynoise and were processed using a noisereduction algorithm that removes a correlated noise component from each channel, based on the signal seen by the other channels of the array (Broguiere et al. 1995). Since the $1.3 \mathrm{~mm}$ continuum map was obtained by combining several smaller maps taken in the dual-beam mapping mode, it is insensitive to structures more extended than the typical size of an individual bolometer map in azimuth, $\sim 4.5^{\prime}$. The possibility of flux loss from dual-beam filtering is inherent with the observational method and cannot be discarded a priori for extended structures. This point was investigated in detail by Motte et al. (1998), who found a negligible impact on compact continuum cores. We note a good agreement between the determinations of column density based on dust and molecular gas, also in warm gas regions where molecular gas depletion is known to be negligible (see e.g. Bacmann et al. 2002). This makes us confident that the flux losses resulting from dual-beam filtering are rather small, except perhaps in the diffuse cloud in the TC15 region.

Calibration was checked against Mars and secondary calibrators at the IRAM 30 m telescope. The weather conditions were good and rather stable during the two observing sessions. The opacity was monitored every hour on average and we found typical zenith opacities between 0.1 and 0.35 . Pointing was checked every hour as the source transits at low elevation at Pico Veleta and corrections were always found to be lower than $3^{\prime \prime}$. The relative calibration was found to be good to within $10 \%$ and the absolute uncertainty is estimated to be about $15 \%$. The final map has a size of approximately $20^{\prime} \times 10^{\prime}$ and the rms is $8 \mathrm{mJy} / 11^{\prime \prime}$ beam over the whole map. Hence, it is sensitive enough to detect at the $3 \sigma$ level gas+dust column densities of $\sim 10^{22} \mathrm{~cm}^{-2}$ per telescope beam.

In order to outline the weak extended dust components in the nebula, we convolved our map with a Gaussian of 20" HPBW. The size of the condensations is estimated taking into account the deconvolution from the main beam and assuming a Gaussian flux distribution. All the results quoted in this paper are based on the non-degraded map with $6^{\prime \prime}$ resolving power. The continuum source properties are derived adopting a dust temperature $T=$ $22 \mathrm{~K}$, a spectral index $\beta=2$ and a dust opacity coefficient $\kappa_{250}=$ $0.1 \mathrm{~cm}^{2} \mathrm{~g}^{-1}$. These parameters were obtained from the analysis of the dust emission in the cores TC2 and TC4 (LCO0; L02).

\subsection{Millimeter line observations}

A region of 10 arcmin size centered on the Trifid was mapped at full sampling in the $\mathrm{CO} J=2 \rightarrow 1, J=1 \rightarrow 0,{ }^{13} \mathrm{CO} J=1 \rightarrow 0$ 
Table 1. Millimeter line observations.

\begin{tabular}{lccccc}
\hline \hline Line & $\begin{array}{c}\text { Frequency } \\
(\mathrm{GHz})\end{array}$ & Telescope & $\begin{array}{c}\text { Beamwidth } \\
(\operatorname{arcsec})\end{array}$ & $B_{\text {eff }}$ & $F_{\text {eff }}$ \\
\hline $\mathrm{HCO}^{+} J=1 \rightarrow 0$ & 89.18852 & IRAM & 28 & 0.77 & 0.90 \\
$\mathrm{CS} J=2 \rightarrow 1$ & 97.98097 & IRAM & 24 & 0.71 & 0.90 \\
& & SEST & 48 & 0.72 & 1.0 \\
$\mathrm{C}^{18} \mathrm{O}=1 \rightarrow 0$ & 109.28218 & IRAM & 22 & 0.68 & 0.92 \\
${ }^{13} \mathrm{CO} J=1 \rightarrow 0$ & 110.20135 & IRAM & 22 & 0.68 & 0.92 \\
${ }^{12} \mathrm{CO} J=1 \rightarrow 0$ & 115.27120 & IRAM & 21 & 0.67 & 0.92 \\
$\mathrm{CS} J=3 \rightarrow 2$ & 146.96905 & IRAM & 16 & 0.53 & 0.90 \\
& & SEST & 32 & 0.66 & 1.0 \\
$\mathrm{CO} J=2 \rightarrow 1$ & 230.53800 & IRAM & 11 & 0.45 & 0.86 \\
$\mathrm{CO} J=3 \rightarrow 2$ & 345.79599 & CSO & 22 & 0.75 & 1.0 \\
\hline
\end{tabular}

and $\mathrm{C}^{18} \mathrm{O} J=1 \rightarrow 0$ lines with the IRAM $30 \mathrm{~m}$ telescope (Pico Veleta, Spain) in July 1996, using the "On-the-Fly" technique. We used both and autocorrelator and $1 \mathrm{MHz}$ filterbanks as spectrometers. The autocorrelator provided a kinematic resolution of $0.2 \mathrm{~km} \mathrm{~s}^{-1}$ for all the transitions. The kinematic resolution provided by the filterbanks ranges from $3.5 \mathrm{~km} \mathrm{~s}^{-1}$ for the $J=1 \rightarrow 0$ transitions of the CO isotopomers to $1.3 \mathrm{~km} \mathrm{~s}^{-1}$ for the $J=2 \rightarrow 1$ transitions. An "intermediate" reference position was taken 10 arcmin East of the Trifid; the molecular emission at this position was later observed in position switching mode, adopting an Off position checked to be free of emission.

A large map of $0.5 \mathrm{deg} \times 0.5 \mathrm{deg}$ was obtained at the CSO in the CO $J=3 \rightarrow 2$ transition during various observing runs between 1998 and 1999, also using the "on-the-fly" technique with a reference position free of emission. The map was sampled at $20^{\prime \prime}$, corresponding to approximately one beamwidth. The receiver was connected to an AOS which provided a kinematic resolution of $0.4 \mathrm{~km} \mathrm{~s}^{-1}$.

Observations of the Trifid in the lines of CO $J=1 \rightarrow 0$, $\mathrm{CS} J=2 \rightarrow 1$ and $J=3 \rightarrow 2$ and $\mathrm{HCO}^{+} J=1 \rightarrow 0$ were obtained with the SEST telescope (La Silla, Chile) in September 1996 and October 1997. The observations were carried out in position switching mode, using the same reference position as for the OTF IRAM data. We observed a total area of $20^{\prime} \times 20^{\prime}$ with a spacing of $20^{\prime \prime}$ over most of the region. Two strips of $2^{\prime} \times 15^{\prime}$ at the eastern and western border of the field, where the molecular emission is much less, were mapped with a $40^{\prime \prime}$ sampling. An AOS was used as a spectrometer, which provided a spectral resolution of $43 \mathrm{kHz}\left(0.11 \mathrm{~km} \mathrm{~s}^{-1}\right.$ at the frequency of the $\mathrm{CO} J=1 \rightarrow 0$ line).

We have adopted the main-beam brightness temperature scale to express the brightness of the lines studied here, with the exception of the ${ }^{12} \mathrm{CO}$ and ${ }^{13} \mathrm{CO}$ lines. As it appears in our observations, bright ${ }^{12} \mathrm{CO}$ and ${ }^{13} \mathrm{CO}$ emission extends over regions of several tens of arcmin. At such scales, the main-beam temperature is no longer a good approximation to the actual line brightness because the error beam contribution is no longer negligible. The antenna temperature corrected for rearward losses $T_{\mathrm{A}}^{*}$ appears as a better estimate and we adopt this scale for the main isotopologue transitions of $\mathrm{CO}$. The validity of this approximation was discussed previously by L02 (see Sect. 7.1). The observed molecular lines, their frequency, the telescope beamwidth, the main-beam efficiency $B_{\text {eff }}$ and the forward efficiency $F_{\text {eff }}$ are summarized in Table 1 .

The physical conditions in the molecular gas associated with the condensations were derived from the millimeter transitions of CS and the CO isotopomers. The kinetic temperature was estimated from the $\mathrm{CO} J=2 \rightarrow 1$ antenna temperature, and found to lie in the range $25-30 \mathrm{~K}$. In some cores, a simple LTE analysis of the ${ }^{13} \mathrm{CO}$ and $\mathrm{C}^{18} \mathrm{O}$ lines could be performed and showed very consistent results with the values derived from the ${ }^{12} \mathrm{CO}$ data. In all the condensations, the ratio of the ${ }^{13} \mathrm{CO} J=1 \rightarrow 0$ to the $\mathrm{C}^{18} \mathrm{O} J=1 \rightarrow 0$ intensities was found to be very close to the canonical abundance ratio (8), within the uncertainties. It implies that the $\mathrm{C}^{18} \mathrm{O}$ line is optically thin, and the ${ }^{13} \mathrm{CO}$ either optically thin or moderately optically thick $(\tau \simeq 1)$. The very good consistency of the results from the different isotopic $\mathrm{CO}$ lines expressed in different units $\left(\mathrm{C}^{18} \mathrm{O}\right.$ intensities are expressed in units of main-beam brightness temperature, unlike ${ }^{12} \mathrm{CO}$ and ${ }^{13} \mathrm{CO}$ ) provides good support to the hypothesis made in our analysis.

In the analysis of the dense gas, as traced by CS, we have adopted a lower value for the kinetic temperature $(20 \mathrm{~K})$, comparable to the cold dust temperature derived from the spectral energy distribution of the cores detected in the survey (see above). The density and column density of the high-density gas were estimated from a simple analysis of the CS line emission in the Large-Velocity Gradient approximation. The linewidth was obtained from a Gaussian fit to the line profiles of the $J=2 \rightarrow 1$ and $J=3 \rightarrow 2$ transitions. We have assumed a standard abundance $[\mathrm{CS}] /\left[\mathrm{H}_{2}\right]=10^{-9}$.

The dynamics of the molecular cloud gas will be discussed in detail on the basis of our molecular line emission survey and presented in a forthcoming paper (Lefloch 2008, in prep.).

\section{Results}

\subsection{Dust filaments}

The presence of "stellar incubators" in M 20 was searched for by mapping the distribution of the cold dust emission at $1.3 \mathrm{~mm}$. We have mapped the emission of the parental cloud over an area of about 15 arcmin in RA by 20 arcmin in declination. The final map is presented in Fig. 1. It offers a very contrasted picture of the nebula with the best angular resolution available in our millimeter wavelengths observations $\left(11^{\prime \prime}\right)$. All the condensations discovered in the survey are distributed in a complex network of filamentary structures. Some of these filaments are detected as dark lanes against the bright nebular background in the optical. The surface layers of the UV-heated filaments in the nebula are detected in the mid-infrared with ISOCAM (CL98) and SPITZER (Rho et al. 2006). Four main filaments could be identified on the front side of M20 (Fig. 1): the lanes which trisect the nebula (southern, eastern, western lanes) and a long filament on the western side (12 pc), detected in absorption in the optical, to which we will refer as the "western filament" (WF; see also Lefloch et al. 2003). Observations of the molecular gas emission has revealed several other filaments of similar length, $10 \mathrm{pc}$ or more, extending far beyond M 20, both in the northern and 

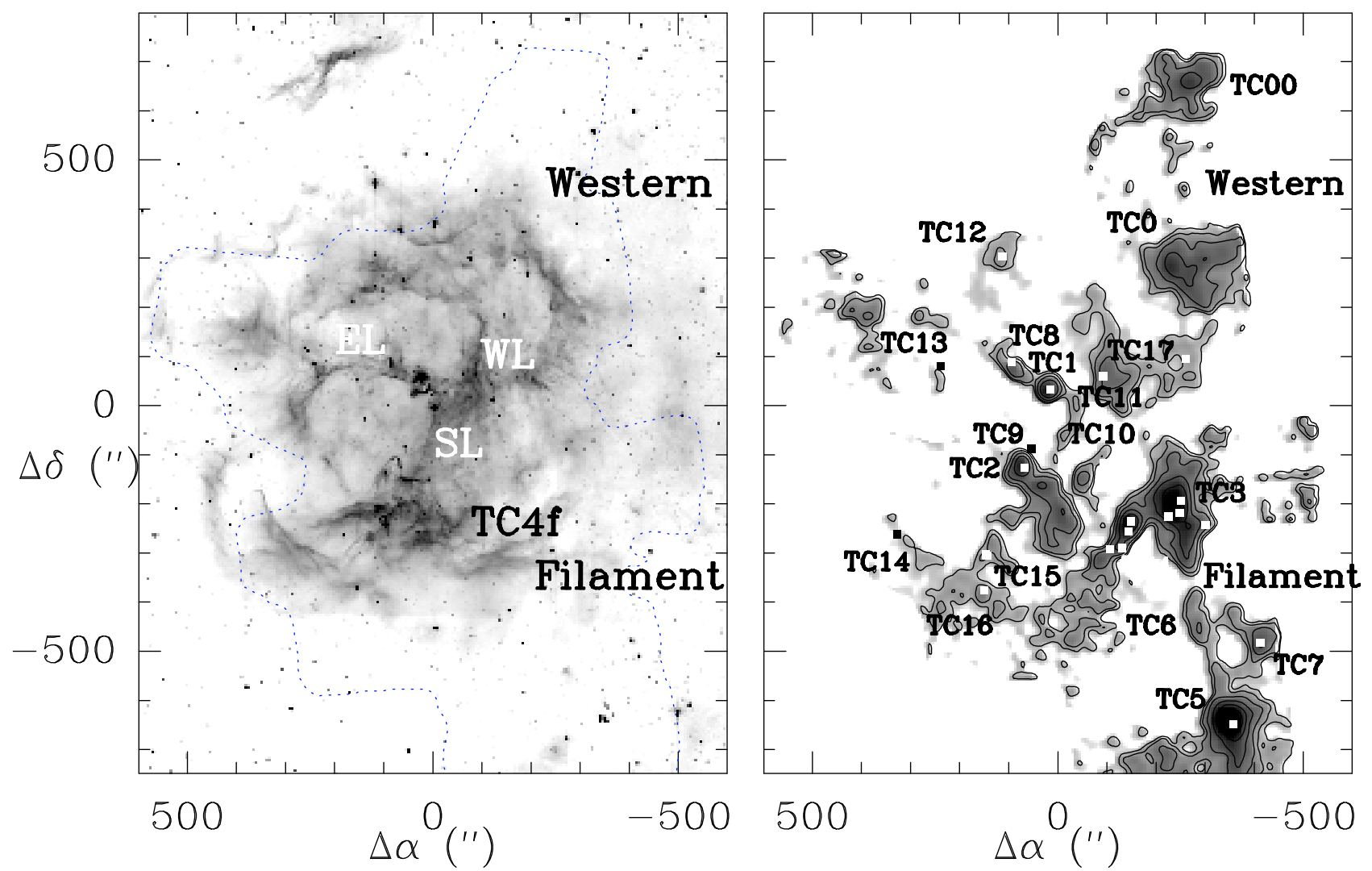

Fig. 1. (Left) Mid-infrared continuum emission at $8 \mu \mathrm{m}$ around M 20 as observed with SPITZER (Rho et al. 2006). The dotted line traces the limit of the field observed with the $1.3 \mathrm{~mm}$ bolometer array. (right) Thermal dust emission at $1300 \mu \mathrm{m}$ around M 20. Contour levels are 5, 10, 60, 120, $240,480 \mathrm{mJy} / 11^{\prime \prime}$ beam. The location of the Class0/I sources identified by SPITZER is marked by squares.

Table 2. Physical properties of the filaments.

\begin{tabular}{lccccccccc}
\hline \hline Name & $\begin{array}{c}\text { Length } \\
\mathrm{pc}\end{array}$ & $\begin{array}{c}\text { Diameter } \\
\mathrm{pc}\end{array}$ & $\begin{array}{c}\sigma \\
\mathrm{km} \mathrm{s}^{-1}\end{array}$ & $\begin{array}{c}\text { Mass } \\
M_{\odot}\end{array}$ & $\begin{array}{c}N(\mathrm{H}) \\
\mathrm{cm}^{-2}\end{array}$ & $\begin{array}{c}\text { Density } \\
\mathrm{cm}^{-3}\end{array}$ & $\begin{array}{c}\text { Fragment sep. } \\
\mathrm{pc}\end{array}$ & $\begin{array}{c}\text { Mass per u.l. } \\
M_{\odot} \mathrm{pc}^{-1}\end{array}$ & $\begin{array}{c}\text { Virial mass } \\
\mathrm{pc}\end{array}$ \\
\hline WF & 11.8 & 1.0 & 0.7 & 2330 & $1.5(22)$ & $2.0 \times 10^{3}$ & 3.6 & 197 & 220 \\
EL & 1.9 & 0.47 & 0.65 & 138 & $2.0(22)$ & $6.9 \times 10^{3}$ & 0.8 & 74 & 190 \\
SL & 1.6 & 0.45 & 0.5 & 66 & $9.0(21)$ & $9.0 \times 10^{3}$ & 0.7 & 39 & 104 \\
TC4f & 1.3 & 0.33 & 0.7 & 237 & $5.5(22)$ & $2.5 \times 10^{4}$ & 0.41 & 177 & 210 \\
\hline
\end{tabular}

$a(b)$ stands for $a \times 10^{b}$.

southern directions, on the rear side of the nebula. The TC4 region was previously characterized as a compressed shocked layer, filamentary-like, of accumulated material Southwest of the nebula (LC00), dubbed "TC4f". Our survey shows that the filament stretches far away from M 20, to a region of diffuse emission, South of the nebula.

The physical properties of the filaments were derived from the millimeter thermal dust emission. The average gas column density $N(\mathrm{H})$ was obtained from the average flux integrated in the region defined by the intensity contour $10 \mathrm{mJy} / 11^{\prime \prime}$ beam and is of the order of a few $10^{22} \mathrm{~cm}^{-2}$. The average density was derived dividing the gas column density by the mean filament diameter, estimated from the contour $10 \mathrm{mJy} / 11^{\prime \prime}$ beam. To ease comparison with models, we also computed the mass, mass per unit length of each filament, and determined the sound speed from the $\mathrm{C}^{18} \mathrm{O}$ line profiles. The physical properties of the filaments are summarized in Table 2. The filaments are rather dense, from a few $10^{3} \mathrm{~cm}^{-3}$ to about $10^{4} \mathrm{~cm}^{-3}$ in the central regions. WF is noticeably larger and more massive than the other filaments, but the molecular gas density and the kinetic energy density are very similar in all of them. The physical properties of WF, such as its size, its mass per unit length, compare very well with those of the Orion filaments (Maddalena et al. 1986) and the infrared dark clouds (IRDCs; see Beuther et al. 2007, for a review).

All the filaments display evidence of fragmentation at small and large scales. WF is fragmented in a long chain of massive condensations which stretches down to the W28 supernova remnant (SNR), located at a distance of $1.9 \mathrm{kpc}$, similar to M 20 (Velázquez et al. 2002). We have estimated the mass of each condensation by integrating on the contour of millimeter continuum emission at $10 \mathrm{mJy} / 11^{\prime \prime}$ beam; we obtain masses of several hundreds $M_{\odot}$ for each condensation (see Table 4; also Sect. 7). Evidence of star formation was previously found in WF, thanks to the discovery of TC3 (LC00). Several water masers were discovered in TC3, one of which is associated with a SPITZER protostellar object (Healy et al. 2004).

The detection of Class $0 / \mathrm{I}$ protostellar sources in TC1 and TC8 testifies that star formation is going on in the eastern lane. The southern lane is an exception as it does not display any hint of star formation activity although two condensations were discovered in the filament. Such non star-forming (pre-stellar) cores play an important role as they bring information on the physical conditions of the parental cloud, before the onset of star 
Table 3. Condensations discovered at millimeter wavelengths near M 20. Star-forming condensations are marked by with a $\uparrow$.

\begin{tabular}{|c|c|c|c|c|c|c|c|c|c|}
\hline Name & $\alpha(2000)$ & $\delta(2000)$ & Dimensions & $\begin{array}{l}\text { Core } \\
\left(M_{\odot}\right)\end{array}$ & $\begin{array}{c}\text { Core virial mass }{ }^{2} \\
\left(M_{\odot}\right)\end{array}$ & $\begin{array}{c}\text { Fragment } \\
\left(M_{\odot}\right)\end{array}$ & $\begin{array}{c}{\text { (Virial })^{1} \text { Mass }}^{\left(M_{\odot}\right)} \\
\end{array}$ & $\begin{array}{l}N(\mathrm{H})^{2} \\
\left(\mathrm{~cm}^{-2}\right)\end{array}$ & \\
\hline TCO0 & 18:01:53:63 & $-22: 50: 57.6$ & $160^{\prime \prime}$ & 49 & $56 / 93$ & 250 & $213 / 355$ & $7.0(22)$ & WF \\
\hline TCO & $18: 02: 06.35$ & $-22: 57: 13.6$ & $180^{\prime \prime} \times 80^{\prime \prime}$ & & & 474 & $196 / 327$ & $9.5(22)$ & WF \\
\hline TCOA & 18:02:06.35 & $-22: 57: 13.6$ & $53^{\prime \prime} \times 24^{\prime \prime}$ & $\overline{49}$ & $58 \overline{8} 97$ & - & 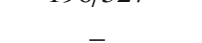 & $9.5(22)$ & WF \\
\hline TCOB & 18:02:01.70 & $-22: 57: 00.0$ & $21^{\prime \prime} \times 9^{\prime \prime}$ & 11 & $15 / 24$ & - & - & $5.7(22)$ & WF \\
\hline TCOC & $18: 02: 03.42$ & $-22: 57: 27.6$ & $17^{\prime \prime} \times 9^{\prime \prime}$ & 8 & $16 / 27$ & - & - & $5.7(22)$ & WF \\
\hline TCOD & 18:02:04.00 & $-22: 57: 55.5$ & $15^{\prime \prime} \times 15^{\prime \prime}$ & 9 & $19 / 33$ & & & $5.2(22)$ & WF \\
\hline $\mathrm{TC} 1 \dagger$ & $18: 02: 24.68$ & $-23: 01: 17.7$ & $46^{\prime \prime} \times 46^{\prime \prime}$ & 28 & $23 / 38$ & $\overline{69}$ & $55 \overline{5 / 91}$ & $1.6(23)$ & $\mathrm{E}$ \\
\hline $\mathrm{TC} 2 \dagger^{4}$ & $18: 02: 28.49$ & $-23: 03: 56.9$ & $50^{\prime \prime} \times 36^{\prime \prime}$ & 27 & $16 / 27$ & 63 & $35 / 59$ & $1.6(23)$ & _- \\
\hline $\mathrm{TC} 3 \dagger^{5}$ & 18:02:05.57 & $-23: 05: 29.0$ & $200^{\prime \prime} \times 110^{\prime \prime}$ & - & - & 750 & $294 / 489$ & $3.7(23)$ & $\overline{\mathrm{WF}}$ \\
\hline $\mathrm{TC} 3 \mathrm{~A} \dagger^{5}$ & $18: 02: 05.74$ & $-23: 05: 32.5$ & $20^{\prime \prime} \times 20^{\prime \prime}$ & 90 & $39 \overline{9} 64$ & - & - & $3.7(23)$ & WF \\
\hline $\mathrm{TC} 3 \mathrm{~B}^{5}$ & 18:02:04.07 & $-23: 06: 06.6$ & $13^{\prime \prime} \times 13^{\prime \prime}$ & 17 & $24 / 42$ & - & - & $1.3(23)$ & WF \\
\hline $\mathrm{TC} 3 \mathrm{C} \uparrow^{5}$ & $18: 02: 05.87$ & $-23: 05: 03.9$ & $15^{\prime \prime} \times 15^{\prime \prime}$ & 34 & $30 / 49$ & & - & $1.9(23)$ & WF \\
\hline $\mathrm{TC} 4$ & $18: 02: 12.77$ & $-23: 05: 46.7$ & $160^{\prime \prime} \times 40^{\prime \prime}$ & . & 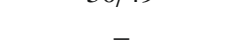 & $2 \overline{3} 7$ & $159 \overline{9} 265$ & $2.8(23)$ & - \\
\hline $\mathrm{TC} 4 \mathrm{~A} \dagger^{5}$ & $18: 02: 12.77$ & $-23: 05: 46.7$ & $32^{\prime \prime} \times 16^{\prime \prime}$ & $\overline{58}$ & $31 \overline{1 / 51}$ & - & - & $2.8(23)$ & - \\
\hline $\mathrm{TC} 4 \mathrm{~B} \dagger^{5}$ & $18: 02: 14.43$ & $-23: 06: 47.5$ & $12^{\prime \prime} \times 12^{\prime \prime}$ & 11 & $16 / 27$ & - & - & $8.6(22)$ & - \\
\hline $\mathrm{TC} 4 \mathrm{C}^{5}$ & $18: 02: 17.00$ & $-23: 07: 12.9$ & $13.5^{\prime \prime} \times 13.5^{\prime \prime}$ & 8 & $18 / 30$ & - & - & $6.5(22)$ & - \\
\hline TC5 & $18: 01: 57.65$ & $-23: 12: 44.6$ & $140^{\prime \prime} \times 80^{\prime \prime}$ & 177 & $134 / 223$ & $8 \overline{07}$ & $850 / \overline{1} 418$ & $9.9(23)$ & $\overline{\mathrm{WF}}$ \\
\hline TC6 & 18:02:03.37 & $-23: 08: 40.6$ & $120^{\prime \prime} \times 50^{\prime \prime}$ & 1. & - & 43 & $126 / 210$ & $3.6(22)$ & WF \\
\hline TC6A & $18: 02: 02.83$ & $-23: 09: 39.2$ & $11^{\prime \prime} \times 11^{\prime \prime}$ & $\overline{3}$ & $18 \overline{3} 30$ & _- & - & $2.9(22)$ & WF \\
\hline TC6B & 18:02:01.94 & $-23: 09: 22.0$ & $11^{\prime \prime} \times 11^{\prime \prime}$ & 2.5 & $18 / 30$ & - & - & $2.5(22)$ & WF \\
\hline TC6C & 18:02:03.36 & $-23: 08: 39.7$ & $11^{\prime \prime} \times 11^{\prime \prime}$ & 2 & $18 / 30$ & - & - & $2.3(22)$ & WF \\
\hline TC6D & 18:02:03.01 & $-23: 08: 25.7$ & $11^{\prime \prime} \times 11^{\prime \prime}$ & 2 & $18 / 30$ & & & $2.0(22)$ & WF \\
\hline $\mathrm{TC} 7+$ & $18: 02: 03.50$ & $-23: 09: 51.2$ & $160^{\prime \prime} \times 100^{\prime \prime}$ & 17 & $39 / 65$ & $\overline{63}$ & $102 \overline{2} 170$ & $8.8(22)$ & WF \\
\hline $\mathrm{TC} 8 \dagger$ & $18: 02: 30.38$ & $-23: 00: 22.2$ & $88^{\prime \prime} \times 34^{\prime \prime}$ & 13 & $14 / 24$ & 46 & $66 / 109$ & $7.0(22)$ & $\mathrm{E}$ \\
\hline $\mathrm{TC} 9 \dagger$ & $18: 02: 27.25$ & $-23: 03: 20.3$ & $17^{\prime \prime} \times 13^{\prime \prime}$ & & & 4 & $7 / 12$ & $1.6(22)$ & - \\
\hline TC10 & $18: 02: 22.3$ & $-23: 02: 56.0$ & $24^{\prime \prime} \times 9^{\prime \prime}$ & 2.5 & $5 / 8$ & 25 & $30 / 51$ & $2.3(22)$ & \\
\hline TC11 & $18: 02: 20.4$ & $-23: 01: 24.0$ & $44^{\prime \prime} \times 28^{\prime \prime}$ & - & - & 6 & $23 / 38$ & $9.0(21)$ & $\overline{\mathrm{S}}$ \\
\hline $\mathrm{TC} 12 \dagger$ & $18: 02: 31.66$ & $-22: 56: 46.2$ & $56^{\prime \prime} \times 32^{\prime \prime}$ & - & - & 9 & $76 / 126$ & $9.1(22)$ & \\
\hline $\mathrm{TC} 13 \dagger$ & $18: 02: 40.73$ & $-23: 00: 30.6$ & $55^{\prime \prime} \times 35^{\prime \prime}$ & - & - & 3.5 & $65 / 108$ & $1.2(22)$ & $\overline{\mathrm{E}}$ \\
\hline $\mathrm{TC} 14 \dagger$ & $18: 02: 47.26$ & $-23: 06: 13.0$ & unres. & - & - & & - & $4.7(21)$ & - \\
\hline $\mathrm{TC} 15 \dagger$ & $18: 02: 34.17$ & $-23: 06: 53.0$ & $21^{\prime \prime} \times 11^{\prime \prime}$ & 1.4 & - & $\overline{4.7}$ & $18 \overline{8 / 30}$ & $9.3(21)$ & - \\
\hline TC16† & $18: 02: 34.46$ & $-23: 08: 06.4$ & $36^{\prime \prime} \times 26^{\prime \prime}$ & 1.8 & - & 9.4 & $24 / 49$ & $1.2(22)$ & - \\
\hline $\mathrm{TC} 17$ & 18:02:16.85 & $-23: 00: 51.8$ & $11^{\prime \prime} \times 11^{\prime \prime}$ & - & - & 7 & $12 / 20$ & $4.4(22)$ & $\overline{\mathrm{W}}$ \\
\hline
\end{tabular}

${ }^{1}$ The virial mass was computed assuming an isothermal sphere with density $\rho \propto r^{-2}: M_{\mathrm{v}}=3 R \sigma^{2} / G$ and uniform density $M_{\mathrm{v}}=5 R \sigma^{2} / G$; the velocity dispersions $\sigma$ is derived from $\mathrm{C}^{18} \mathrm{O}$ line observations, or ${ }^{13} \mathrm{CO}$ if $\mathrm{C}^{18} \mathrm{O}$ is not detected.

${ }^{2}$ Peak column density. ${ }^{3} \mathrm{WF}=$ Western filament; $\mathrm{E}=$ Eastern Lane; $\mathrm{W}=$ Western Lane; $\mathrm{S}=$ Southern Lane. ${ }^{4}$ L02. ${ }^{5}$ LC00.

formation. Unfortunately, detailed molecular observations are available only for a few of them (TC0, TC6, TC10, TC16).

\subsection{Dust cores in $M 20$}

The continuum sources and their physical properties (size, mass, column density) are listed in Table 3 . The sources can be divided into two categories:

- condensations (or massive fragments) with a typical size of $100^{\prime \prime}(1 \mathrm{pc})$ and a mass of several hundred $M_{\odot}$. Their extent is defined by the contour at $10 \mathrm{mJy} / 11^{\prime \prime}$ beam;

- cores with a typical size of a few $10^{\prime \prime}(0.1 \mathrm{pc})$ and a mass of a few ten of $M_{\odot}$. They are subunits of the fragment and their extent is defined by the contour at half power.

All the cores detected in our survey have a typical size in the range $10^{\prime \prime}-20^{\prime \prime}(0.1-0.2 \mathrm{pc})$. The gas density at the continuum emission peak ranges between $10^{5} \mathrm{~cm}^{-3}$ and a few $10^{6} \mathrm{~cm}^{-3}$. The mean core density is higher by a factor of 10 , typically, in the regions where evidence of shock has been detected.

Of the 33 condensations identified in our survey and listed in Table 1, 16 sources are associated with SPITZER protostellar candidates (Rho et al. 2006). Most of the young stellar objects identified by SPITZER are not associated with large amount of cold molecular gas or cold dust emission in our survey $\left(N\left(\mathrm{H}_{2}\right) \geq 10^{22} \mathrm{~cm}^{-2}\right)$. This is the case of TC14, for which we failed to detect any associated continuum or line emission and the case of the young stellar objects IRS 6 and IRS 7 discovered by L01; they exhibit the $9.7 \mu \mathrm{m}$ silicate band in emission, a direct evidence of the weak amount of parental material still present. We have searched for outflow signatures towards the protostar candidates. In most of the cases, confusion from the ambient molecular cloud is such that it is not possible to discriminate the outflow wing emission in tracers as common as CO. It is necessary to observe tracers with a more constrasted emission, hence less abundant, to detect the signature of the outflowing gas. Unfortunately, it becomes more difficult to detect this emission in low-density gas region, such as the cometary globules located on the eastern side of M20. In a few sources, however, we detected the high-velocity wings of protostellar outflows in the CS and SiO line profiles (TC3, TC4, TC5). We also detected a protostellar Herbig-Haro jet associated with the protostellar core TC2 (CL98; Rosado et al. 1999; L02).

\section{The western filament}

Our extended map shows protostellar condensations TC5, TC7 in addition to the source TC3, previously detected by CL98 and 
Table 4. Physical properties of the cores in the parental cloud of the Trifid.

\begin{tabular}{lcccccccc}
\hline \hline Name & Emission peak & $\begin{array}{c}T k \\
(\mathrm{~K})\end{array}$ & $\begin{array}{r}N\left(\mathrm{H}_{2}\right)^{1} \\
\left(\mathrm{~cm}^{-2}\right)\end{array}$ & $\begin{array}{c}N\left({ }^{13} \mathrm{CO}\right) \\
\left(\mathrm{cm}^{-2}\right)\end{array}$ & $\begin{array}{c}N(\mathrm{CS}) \\
\left(\mathrm{cm}^{-2}\right)\end{array}$ & $\begin{array}{c}n\left(\mathrm{H}_{2}\right)^{2} \\
\left(\mathrm{~cm}^{-2}\right)\end{array}$ & $\begin{array}{c}\text { Mcore } \\
\left(M_{\odot}\right)\end{array}$ & Location \\
\hline TC0A & $\left(-238^{\prime \prime},+294^{\prime \prime}\right)$ & 25 & $4.9(22)$ & $5.5(16)$ & $1.8(13)$ & $5.5(5)$ & 49 & WF \\
TC0B & $\left(-302^{\prime \prime},+292^{\prime \prime}\right)$ & 25 & $2.9(22)$ & $4.0(16)$ & $1.0(13)$ & $4.0(5)$ & 11 & WF \\
TC0C & $\left(-268^{\prime \prime},+275^{\prime \prime}\right)$ & 25 & $2.9(22)$ & $4.5(16)$ & $1.1(13)$ & $4.0(5)$ & 8 & WF \\
TC0D & $\left(-270^{\prime \prime},+252^{\prime \prime}\right)$ & 25 & $2.9(22)$ & $3.8(16)$ & $6.0(12)$ & $4.0(5)$ & 9 & WF \\
TC1 & $\left(+16^{\prime \prime},+33^{\prime \prime}\right)$ & 30 & $8.4(22)$ & $3.6(22)$ & $1.9(13)$ & $3.0(5)$ & 28 & EL \\
TC2 $\dagger$ & $\left(+72^{\prime \prime},-120^{\prime \prime}\right)$ & 30 & $8.0(22)$ & $4.0(16)$ & $1.8(13)$ & $3.0(5)$ & 27 & South \\
TC3A $\$$ & $\left(-246^{\prime \prime},-220^{\prime \prime}\right)$ & 20 & $1.9(23)$ & $6.0(16)$ & $2.0(13)$ & $1.6(6)$ & 90 & WF \\
TC4A $\neq$ & $\left(-150^{\prime \prime},-246^{\prime \prime}\right)$ & 20 & $1.4(23)$ & $6.0(16)$ & $3.3(13)$ & $6.0(5)$ & 58 & WF \\
TC5 & $\left(-360^{\prime \prime},-640^{\prime \prime}\right)$ & 20 & $5.4(23)$ & - & $1.0(14)$ & $2.3(6)$ & 177 & WF \\
TC6 & $\left(-280^{\prime \prime},-440^{\prime \prime}\right)$ & 30 & $1.3(22)$ & $1.5(16)$ & $1.2(13)$ & $8.0(4)$ & $2-3$ & WF \\
TC8 & $\left(+94^{\prime \prime},+87^{\prime \prime}\right)$ & 30 & $3.5(22)$ & $5.1(16)$ & $1.5(13)$ & $4.0(5)$ & 6 & EL \\
TC9 & $\left(+50^{\prime \prime},-90^{\prime \prime}\right)$ & 30 & $\leq 1.0(22)$ & $1.6(16)$ & - & $3.0(4)^{1}$ & 4 & South \\
TC10 & $\left(-20^{\prime \prime},-66^{\prime \prime}\right)$ & 25 & $1.2(22)$ & $2.4(16)$ & $1.5(13)$ & $2.5(5)$ & 25 & SL \\
TC11 & $\left(-42^{\prime \prime},+30^{\prime \prime}\right)$ & 25 & & $1.4(16)$ & - & $1.0(4)^{1}$ & 6 & SL \\
TC13 & $\left(+240^{\prime \prime},+70^{\prime \prime}\right)$ & 25 & $6.0(21)$ & $1.0(16)$ & $6.0(12)$ & $1.0(5)$ & 3.5 & EL \\
\hline
\end{tabular}

${ }^{1}$ Determined from dust; ${ }^{2}$ determined from CS; ${ }^{4}$ Lefloch et al. (2008).

$\dagger$ L02. $\ddagger$ LCO0.

prestellar condensations TC00, TC0, TC6, distributed in a chain of fragments regularly spaced along WF. The SPITZER image of the region (Rho et al. 2006) shows that small clusters of a few protostars have formed in the massive gas fragments TC3 and TC5. In this section, we present a detailed analysis of the condensation TC0. The properties of TC5 and TC6 are discussed in Appendix A.

Condensation TC0 is located in the Northwest of the Trifid at the border of the HII region (Figs. 1, 2). In the optical, it is detected as a region of large obscuration against the diffuse background emission (see e.g. Rho et al. 2006). This shows that the fragment is located on the front side of the nebula, which is consistent with the velocity of the main body gas $v_{\text {lsr }}=+10.2 \mathrm{~km} \mathrm{~s}^{-1}$. Dust emission in the mid-infrared shows the photo-dissociation region as a bright straight bar, with a sharp border at the interface with the HII region (Fig. 2). The molecular gas tracers confirm the presence of such a strong gradient in the emission.

The cold dust emission at $1.3 \mathrm{~mm}$ reveals a condensation with typical dimensions of $100^{\prime \prime} \times 80^{\prime \prime}$ (Fig. 3). The morphology is quite complex and cannot be easily fit by an ellipse, unlike the other dust condensations identified in our survey. The contour at half-power delineates four cores, TCOA-0D (Fig. 3). The "brightest" one, TCOA, is noticeably larger than the other condensations, by a factor of 2 typically. Although the other cores have a comparable brightness, their smaller sizes result in much smaller masses (typically a factor 4). TC0A-0C-0D are located right ahead of the ionization front and the photon-dominated region (PDR), as traced by the $8 \mu \mathrm{m}$ PAH band. By contrast, the core TCOB is further away from the PDR and appears to be better protected from the high-energy radiation.

The physical conditions in the pre-stellar cores TCOA-OB0C-0D are very similar, with column densities $N(\mathrm{CS}) \sim 1.0 \times$ $10^{13} \mathrm{~cm}^{-2}$ and gas densities $n\left(\mathrm{H}_{2}\right) \simeq 4-5 \times 10^{5} \mathrm{~cm}^{-3}$. No evidence for outflow emission was found either in low- and highdensity gas tracers, like in TC3-TC4, which is consistent with the absence of protostellar activity (see Fig. 4). Line profiles towards $0 \mathrm{~B}, 0 \mathrm{C}$, and $\mathrm{OD}$ are doubly peaked. In addition to the main body gas emission, two velocity components are detected, between $+7 \mathrm{~km} \mathrm{~s}^{-1}$ and $+9.2 \mathrm{~km} \mathrm{~s}^{-1}$, and between $+11 \mathrm{~km} \mathrm{~s}^{-1}$ and $+12.5 \mathrm{~km} \mathrm{~s}^{-1}$. In particular the kinematic component at "blueshifted" velocities is detected in the CS line profiles, either

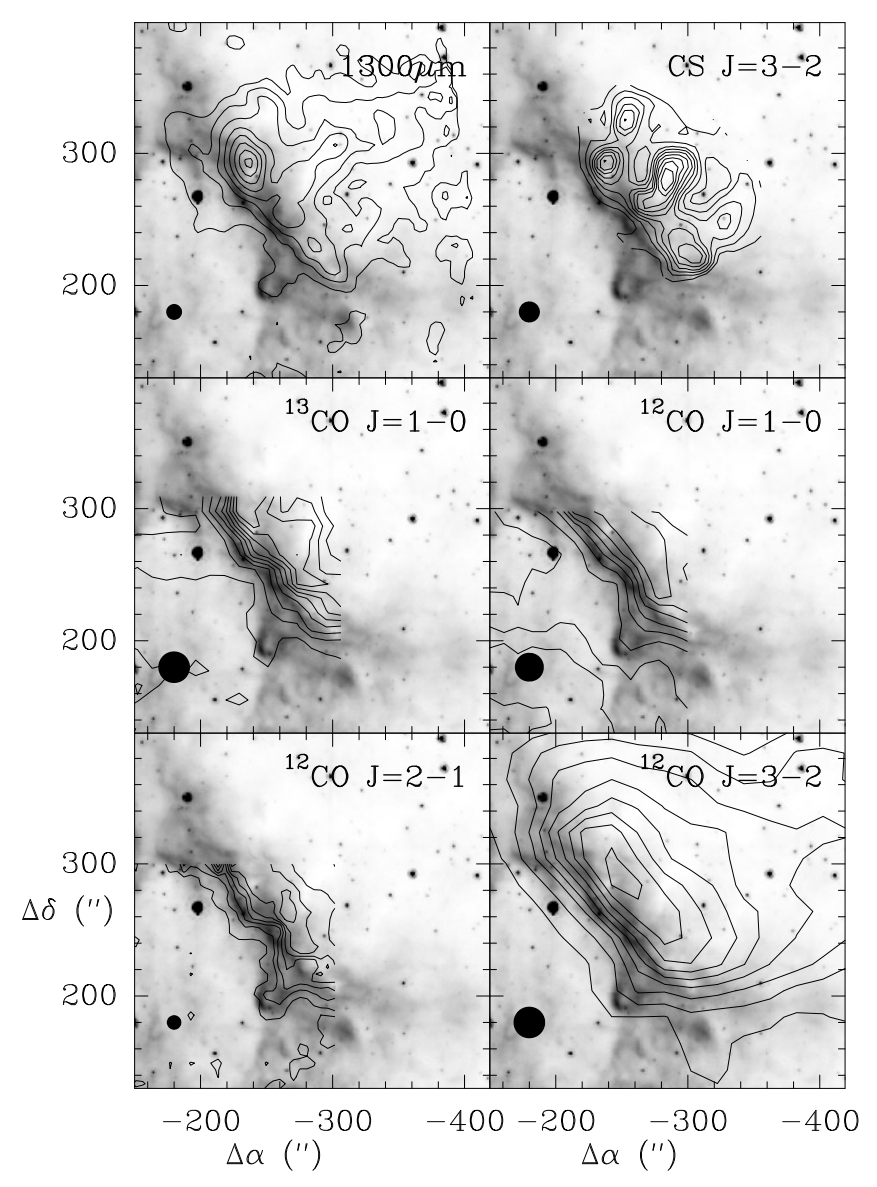

Fig. 2. Millimeter continuum and line emission observed towards TC0 (contours), superposed on a $8 \mu \mathrm{m}$ image of M20 obtained with SPITZER. In the lower left corner of each panel is drawn the beam solid angle (HPFW) of the observations. For each molecular line, the emission is integrated between 6.5 and $13.5 \mathrm{~km} \mathrm{~s}^{-1}$.

as well separated component or as shoulder to the main body line profile. As can be seen in Fig. 5, both secondary components follow closely the border of the PDR excited by M 20, and most likely trace the signature of the shock driven by the ionization front at the surface of the fragment. The widespread 


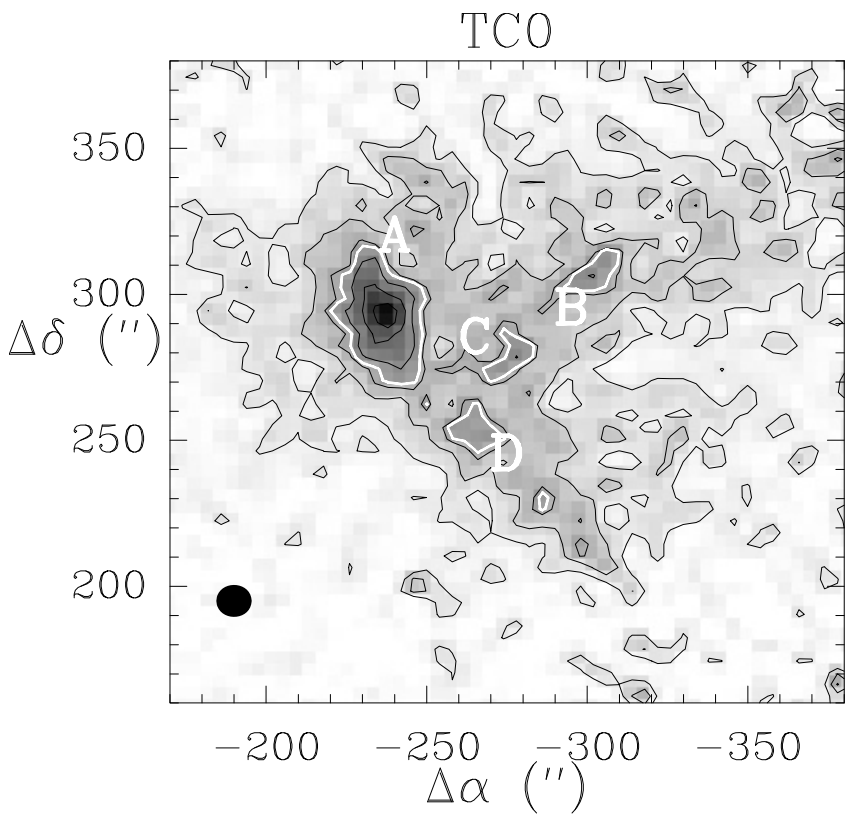

Fig. 3. Thermal dust emission map of TC 0 at $1.3 \mathrm{~mm}$ observed with the IRAM $30 \mathrm{~m}$ telescope. First contour and contour interval are 30 and $15 \mathrm{mJy} / 11^{\prime \prime}$ beam respectively.
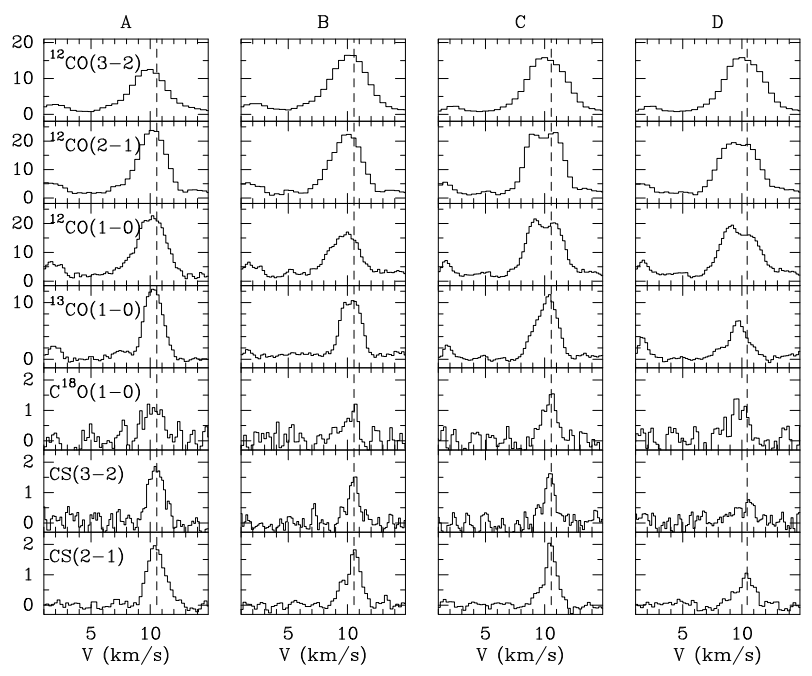

Fig. 4. Molecular line emission observed towards the cores 0A0B-0C-0D of condensation TC0 at offset position $\left(-238^{\prime \prime},+294^{\prime \prime}\right)$, $\left(-302^{\prime \prime},+292^{\prime \prime}\right),\left(-268^{\prime \prime},+264^{\prime \prime}\right)$ and $\left(-270^{\prime \prime},+252^{\prime \prime}\right)$, respectively. Fluxes are expressed in units of antenna temperature. The dashed line marks the velocity of the main body gas.

CS emission detected all over TC0 shows evidence for high density gas $\left(n\left(\mathrm{H}_{2}\right) \approx 10^{5} \mathrm{~cm}^{-3}\right.$.

\section{The eastern lane}

Three main condensations were identified in the eastern lane: TC1, located close to the central stellar cluster (Fig. 6), TC8 and TC13, which both lay further East in the lane (Fig. 7). A fourth, starless, condensation was discovered in the filament, peaking at $\left(-50^{\prime \prime},-10^{\prime \prime}\right)$. We do not report on its properties in detail as it remains undetected in our millimeter continuum survey; we mention it for completeness. TC8 and TC13 are discussed in Appendix B. We discuss in greater detail the properties of TC1, which is the only cometary globule of the sample for which midinfrared spectroscopy could be obtained.

TC 1 appears as a bright condensation in the eastern lane, $40^{\prime \prime}$ East of HD 164492 A (see Figs. 6, 7), both in the millimeter continuum and in the molecular gas emission. The condensation is well separated from the diffuse dust lane emission. The contour at half power defines a central dense core of diameter of $0.16 \mathrm{pc}$ and typical density $n\left(\mathrm{H}_{2}\right)=1.8 \times 10^{5} \mathrm{~cm}^{-3}$. The outer region, with a diameter of $0.4 \mathrm{pc}$ is characterized by lower densities $\left(n\left(\mathrm{H}_{2}\right)=2.5 \times 10^{4} \mathrm{~cm}^{-3}\right)$. The physical parameters of TC1 (gas column density, mass, size, density) are very similar to those derived for the protostellar condensation TC2 in the South of the Trifid (see Table 4 and L02).

The emission of the optical bright rim on the southern border of TC 1 in the optical image of the Trifid (Fig. 6), as well as the detection of the [NeII] line over the globule (Fig. 8) testifies to the condensation being photoionized. The dark appearance of the lanes implies that they lie on the front side of the ionized nebula, and hence they are illuminated on the rear side. The CVF imaging of the UIBs traces the outer parts of the condensation, which looks like an almost continuous ring (Fig. 8). The low emissivity of the central regions can be attributed to absorption by the cold core detected at millimeter wavelengths where large dust and gas column densities are measured, corresponding to $A_{\mathrm{v}} \sim 145$, and $\tau_{12.7 \mu \mathrm{m}}=2$, adopting the reddening law derived by Lynds et al. (1985). We have searched for a mid-IR signature of the protostar discovered in TC1 by integrating the ISOCAM flux between 13.3 and $16.2 \mu \mathrm{m}$, where the radiated flux is the highest in the CVF band, and the extinction, longwards of the silicate absorption range, is the weakest. The images of the globule in the IRAC and ISO LW10 filter are displayed in Fig. 6. A point source is detected very close (within $4^{\prime \prime}$ ) to the cold dust peak (see arrow in Fig. 6). The high-sensitivity SPITZER/IRAC images have detected emission from the source at shorter wavelengths. It is unresolved in these observations $\left(1^{\prime \prime}-2^{\prime \prime}\right)$. A SED was built using the IRAC colors and the MIPS $24 \mu \mathrm{m}$ data (see Rho et al. 2006), which yields an estimate of $570 L_{\odot}$ for the source luminosity. This value is typical of an intermediate-mass protostar.

A spectrum of the source between 5 and $16 \mu \mathrm{m}$ was obtained with ISOCAM/CVF after subtracting the emission of the condensation averaged over the closest neighbouring pixels. Instrumental problems have affected the long wavelength border of the spectrum, resulting in a spurious decrease of the flux beyond $16 \mu \mathrm{m}$. The spectrum in the range $5.0-15.5 \mu \mathrm{m}$ is shown in Fig. 9. The spectrum exhibits a deep absorption due to the silicate band at $9.7 \mu \mathrm{m}$ and a rising slope longward of $10 \mu \mathrm{m}$, characteristic of embedded sources. Longward of $15 \mu \mathrm{m}$, the spectrum displays another dip, due to absorption by the $\mathrm{CO}_{2}$ ice band, centered at $15.2 \mu \mathrm{m}$. The $7.7 \mu \mathrm{m}$ emission band detected in the TC1 spectrum is probably due to PAHs excited on the rear side of the condensation, as was reported in TC2 (L02). The spectrum could be approximately fit by two blackbodies at temperatures of $250 \mathrm{~K}$ and $140 \mathrm{~K}$ modified by dust opacity law $\tau_{v} \propto v^{1.3}$, a spectral index value typical in the mid-IR regime, surrounded by an absorbing cold layer of column density $N(\mathrm{H})=8.0 \times 10^{22} \mathrm{~cm}^{-2}$, and a total $\mathrm{CO}_{2}$ ice column density $N\left(\mathrm{CO}_{2}\right)=3.3 \times 10^{18} \mathrm{~cm}^{-2}$, using integrated absorption cross sections measured in the laboratory (see Cernicharo et al. 2000).

In the absence of observations resolving the emission region, we have assumed the following sizes: 0.2" (350 AU) for the $250 \mathrm{~K}$ component, comparable to the diameter of the disks detected in the central stellar cluster (see L01), 0.5" (800 AU) for the lower temperature component $(140 \mathrm{~K})$. Under such 


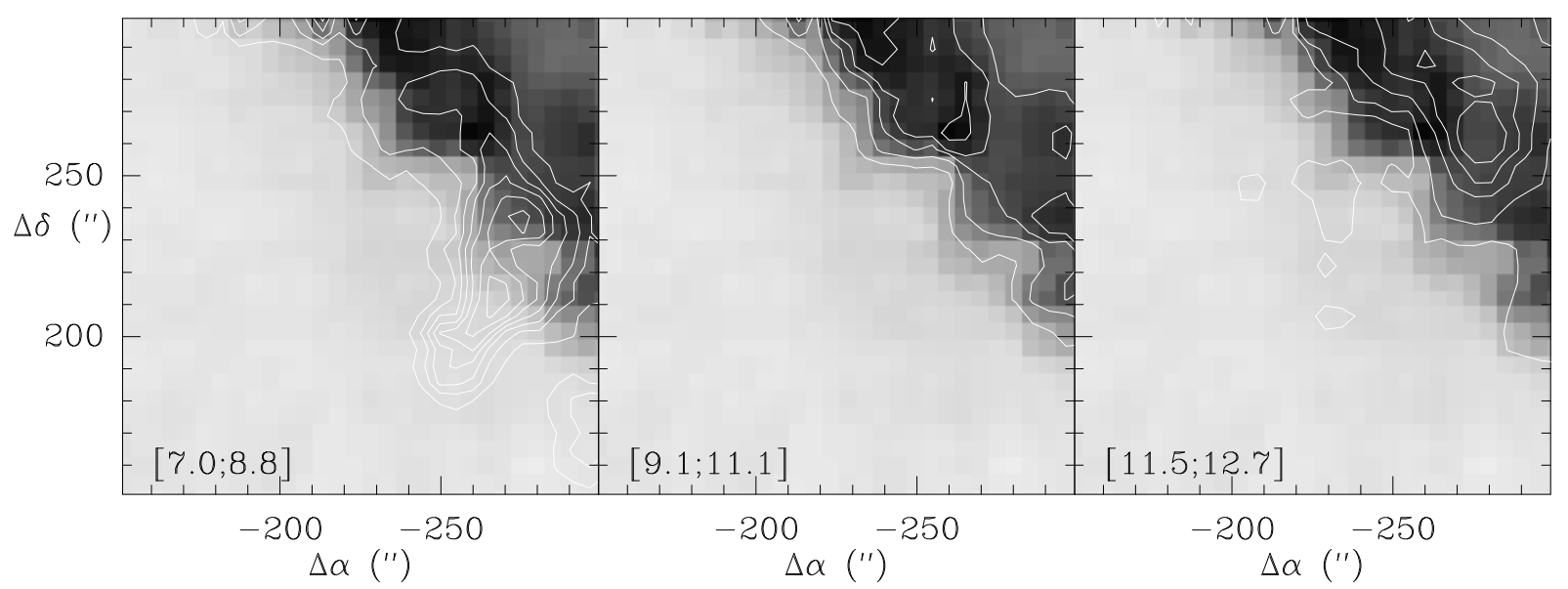

Fig. 5. Emission (contours) of the $\mathrm{CO} J=2 \rightarrow 1$ line integrated in the velocity intervals: $\left[7.0 ; 8.8 \mathrm{~km} \mathrm{~s}^{-1}\right]($ left $),\left[9.1 ; 11.1 \mathrm{~km} \mathrm{~s}^{-1}\right]$ (middle), $\left[11.5 ; 12.7 \mathrm{~km} \mathrm{~s}^{-1}\right]$ (right). The emission is superposed on the emission of the main body gas (greyscale), integrated in the interval $\left[9.1 ; 11.1 \mathrm{~km} \mathrm{~s}^{-1}\right.$ ]. Intensity contours are respectively: $8,11,14, \ldots, 29 \mathrm{~K} \mathrm{~km} \mathrm{~s}^{-1}$ (left); 20, 28, 36, 40, $44 \mathrm{~K} \mathrm{~km} \mathrm{~s}^{-1}$ (middle), 4, 5, 7, 9, 11 K km s (right).

hypothesis, we estimate gas column densities $N(\mathrm{H})=4.0 \times$ $10^{20} \mathrm{~cm}^{-2}$ and $N(\mathrm{H})=3.7 \times 10^{21} \mathrm{~cm}^{-2}$, respectively. The spectrum is thus consistent with the picture of a protostar forming at the center of the TC1 core.

Some residual emission arises in the UIBs at 6.2 and $7.7 \mu \mathrm{m}$. This is reminiscent of the situation in the core of star forming globule TC 2, in which no emission at all is detected between 5 and $17 \mu \mathrm{m}$ apart from a weak emission peak at the position of the $7.7 \mu \mathrm{m}$ UIB. A simple modelling showed that this emission arises from the PDR on the rear side of the globule, where photoionization takes place. It is strongly absorbed by the material of the globule, except near 6.2 and $7.7 \mu \mathrm{m}$ where local minima in the dust absorption curve allow some mid-IR photons to escape. Hence the ring configuration arises naturally if, just like TC2, the rear side of TC1 undergoes heavy photoionization, once the surrounding lower-density material has been photoevaporated. From the absorption produced by the cold dust in the PAH $6.2 \mu \mathrm{m}$ band and the [NeII] line, we derive $\tau_{12.7 \mu \mathrm{m}} \simeq 1.7$ and $\tau_{6 \mu \mathrm{m}}>2.5$. Those values are consistent with the extinction derived from the millimeter continuum observations and an opacity law $\tau_{v} \propto v^{1.3}$.

A secondary component, detected at $v_{\mathrm{lsr}}=+1.8 \mathrm{~km} \mathrm{~s}^{-1}$ could trace the kinematic signature of the shock front driven by the photoionization of the surface layers.

\section{The southern region}

Cometary globule TC2 is the first (proto)stellar incubator identified in the Trifid thanks to the splendid photoionized jet HH 399 which propagates out of the head of the globule (see Fig. 12; also Cernicharo et al. 1998). The dust and molecular line emission of cometary globule TC2, as observed in the mid-infrared with ISO and at millimeter wavelengths, was discussed in detail by L02. The signature of the radiatively-driven implosion of the globule was unambiguously detected in the high-density gas tracers emission $\left(\mathrm{CS}, \mathrm{HCO}^{+}\right)$; numerical modelling of the globule yielded a duration of $0.3 \mathrm{Myr}$ for the photoionization. It comes out that the lifetime of the globule exposed to photoionization is about 1.9 Myr, long enough to allow the protostar to complete the accretion phase.

The ISO data were not sensitive enough to detect emission from the protostar. Based on the SED obtained with ISO

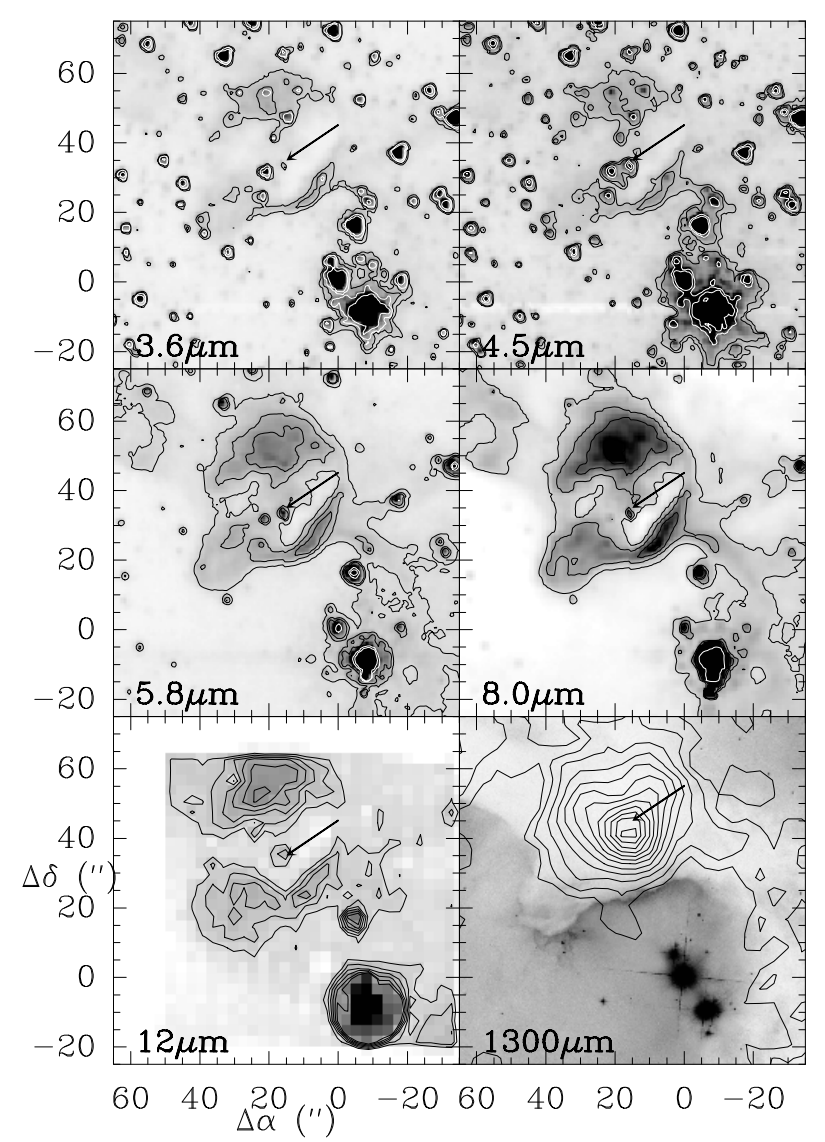

Fig. 6. Cometary globule TC1 as seen in the mid-IR with ISOCAM $(8-15 \mu \mathrm{m})$ and SPITZER $(3.6 \mu \mathrm{m}, 4.5 \mu \mathrm{m}, 5.8 \mu \mathrm{m}, 8.5 \mu \mathrm{m})$. In the bottom right panel the $1.3 \mathrm{~mm}$ thermal dust emission (black contours) superposed on a greyscale image of the $13.3-16.2 \mu \mathrm{m}$, as observed with ISOCAM using the Circular Variable Filter. Contours of cold dust emission are $10,20,40,60$ to $160 \mathrm{mJy} / 11^{\prime \prime}$ beam by step of $20 \mathrm{mJy} / 11^{\prime \prime}$ beam.Contours of mid-IR emission range from 0.25 to $0.65 \mathrm{mJy} / \mathrm{pixel}$ by step of $0.1 \mathrm{mJy} / \mathrm{pixel}$. The arrow points to the position of the protostellar source.

in the range 50-200 $\mu \mathrm{m}$, L02 estimated a source luminosity $L \sim 500 L_{\odot}$, from which they concluded the protostar was an intermediate-mass candidate. Rho et al. (2006) obtained 


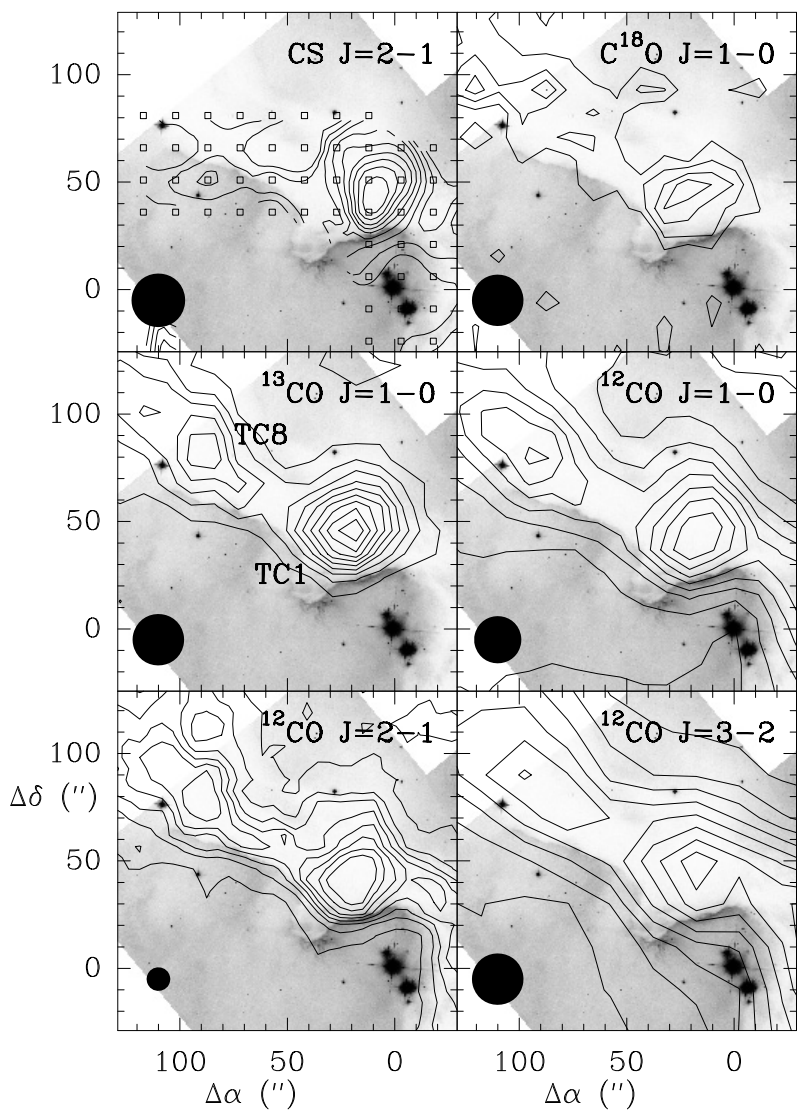

Fig. 7. Millimeter line emission observed in the eastern lane towards TC1 and TC8. The emission is integrated between 0 and $+7 \mathrm{~km} \mathrm{~s}^{-1}$. In the lower left corner of each panel is drawn the beam solid angle (HPFW) of the observations. The line emission integrated between 0 and $7 \mathrm{~km} \mathrm{~s}^{-1}$ is shown in the other panels. For the ${ }^{13} \mathrm{CO}$ and the ${ }^{12} \mathrm{CO}$ transitions the first contour and contour interval are 0.1 times the flux peak value. For the CS $J=2 \rightarrow 1$ line, first contour and contour interval are $0.5 \mathrm{~K} \mathrm{~km} \mathrm{~s}^{-1}$. For the $\mathrm{C}^{18} \mathrm{O}$ emission, the first contour and contour interval are 0.2 times the flux peak value. The positions observed at IRAM are indicated (open squares) in the upper left panel.

a similar estimate $\left(590 L_{\odot}\right)$ using an $3.6-25 \mu \mathrm{m}$ SED constructed from the IRAC and MIPS colors. The protostar powering the jet was only tentatively identified, based on VLA observations of the free-free continuum. The much higher sensitivity of the IRAC images has allowed to detect a few sources with colors typical of young stellar objects, in the region around cometary globule TC2 (see Rho et al. 2006). SPITZER detected only one source with protostellar colors inside the globule. Interestingly, this source is aligned in the HH 399 jet direction, and lies close to the cold dust emission peak (Fig. 12). It coincides with one of the sources marginally detected at the VLA (L02). The source detected with SPITZER/IRAC is most likely the protostar powering HH 399.

ISOCAM detected emission between 8 and $15 \mu \mathrm{m}$ from a small condensation $\left(13^{\prime \prime} \times 17^{\prime \prime}\right)$ in the Northwest of TC2 at offset position (Fig. 12). The condensation is not centered on the flux peak, which indicates the presence of a second, fainter, source. The SPITZER/IRAC images at shorter wavelength indeed unveil two sources located at $\left(50.7^{\prime \prime},-89.2^{\prime \prime}\right)$ and $\left(49.6^{\prime \prime},-97.4^{\prime \prime}\right)$, respectively, lying in a common envelope of $8^{\prime \prime} \times 17^{\prime \prime}$. The two sources have very different IRAC colors. The Northern source is identified as a Class I, with IRAC fluxes similar to those measured towards TC4A, hence compatible with the birth of an
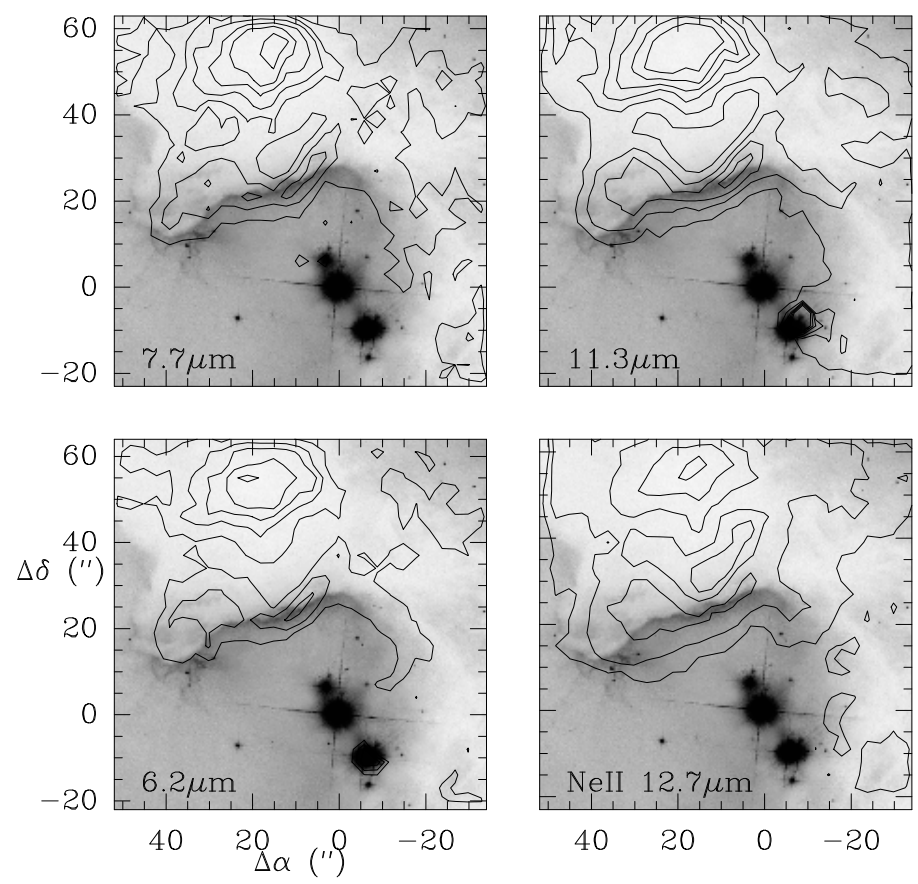

Fig. 8. Spatial distribution of the UIBs at $6.2,7.7,11.3 \mu \mathrm{m}$, and the [NeII] line in the TC 1 region (contours) superposed on an optical $\mathrm{H} \alpha$ image of the region (HST). First contour and contour interval are $0.04 \mathrm{Jy} / \mathrm{px}$ and $0.03 \mathrm{Jy} / \mathrm{px}$ respectively.

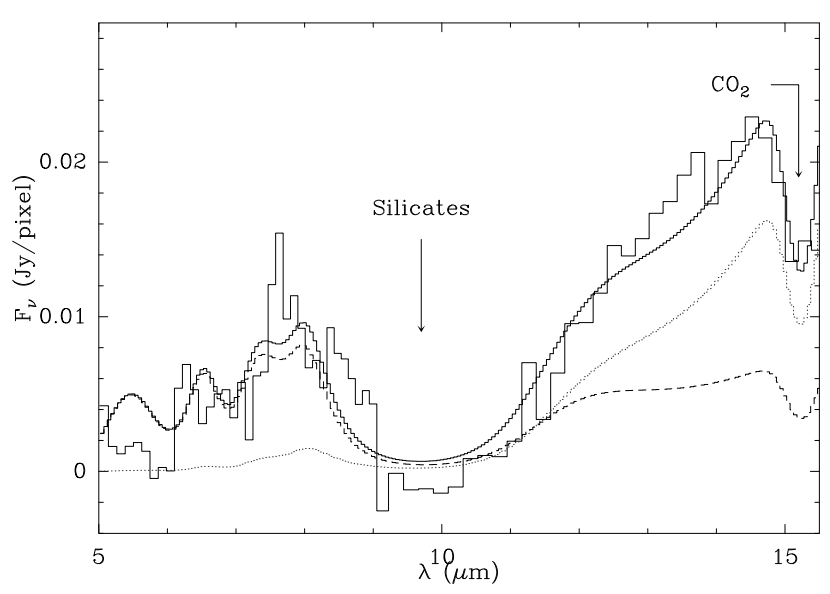

Fig. 9. Spectrum in the range $5-17 \mu \mathrm{m}$ of the protostar embedded in TC 1 (thick), after subtracting the ambient gas component. The spectrum is fit by two black-bodies modified by a dust opacity law $\tau_{v} \propto v^{1.3}$ at $250 \mathrm{~K}$ (dashed) and $140 \mathrm{~K}$ (dotted) respectively. The total fit is displayed in thick.

intermediate-mass object. The southern source has typical stellar colors (Rho et al. 2006). It could be a bright field star, located behind the small dust core, or a young star which has emerged from the photoevaporated layers, like the EGGs of the Elephant trunks in M16 (Hester et al. 1996).

Mid-infrared spectroscopy in the range 5-17 $\mu \mathrm{m}$ was obtained with the CVF. The pixel size was $6^{\prime \prime}$. We present here a spectrum of the emission averaged over the condensation (Fig. 13), after subtracting the emission of a reference position, chosen 1 arcmin North of TC9. The spectrum is dominated by continuum emission decreasing between 5 and $16.5 \mu \mathrm{m}$, and a strong absorption in the $9.7 \mu \mathrm{m}$ silicate band. This shows evidence for a high temperature region inside the object. The overall emission can be roughly reproduced by a blackbody law at a temperature of $250-400 \mathrm{~K}$ modified by a dust 
$\mathrm{TC} 1$

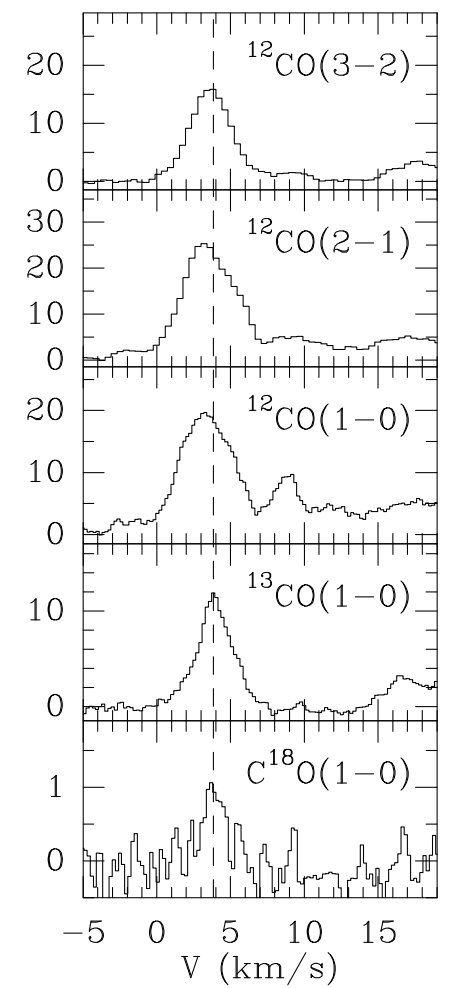

TC8

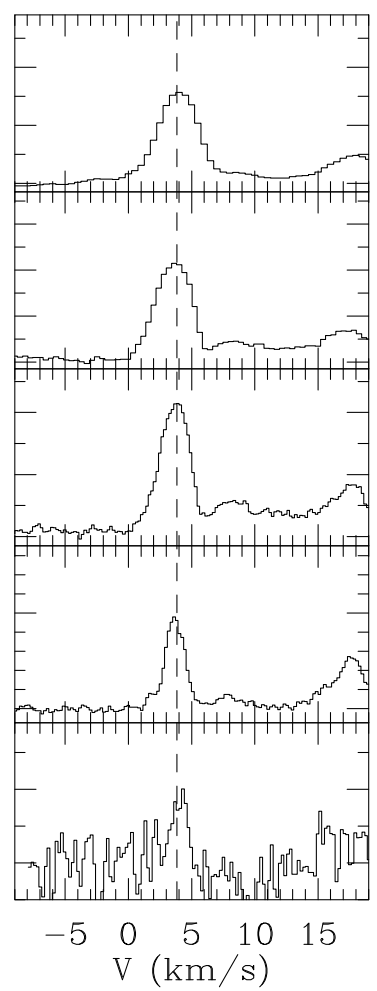

Fig. 10. Spectra obtained towards $\mathrm{TC} 1$ and $\mathrm{TC} 8$ in the transitions $\mathrm{C}^{18} \mathrm{O} J=1 \rightarrow 0,{ }^{13} \mathrm{CO} J=1 \rightarrow 0,{ }^{12} \mathrm{CO} J=1 \rightarrow 0, J=2 \rightarrow 1$ and $J=3 \rightarrow 2$ (from bottom to top). Flux is expressed in units of antenna temperature. The dashed line marks the velocity of the main body gas.

$\mathrm{TC} 1$

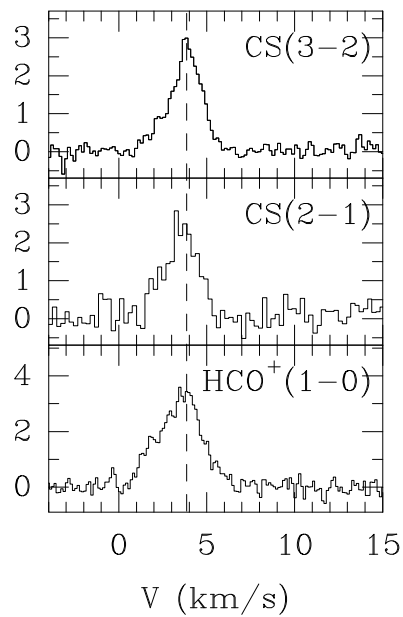

TC8

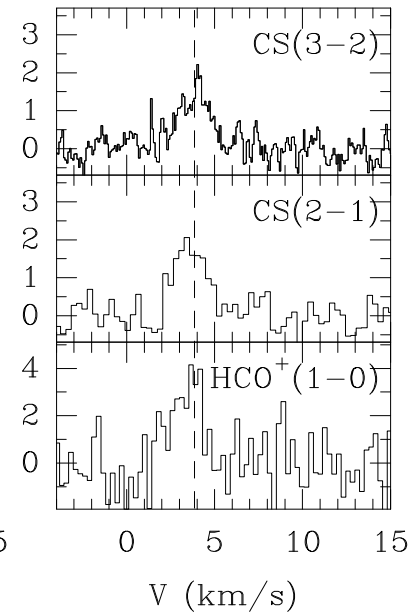

Fig. 11. Dense gas emission observed in the lines of CS $J=2 \rightarrow 1$ and $\mathrm{HCO}^{+} J=1 \rightarrow 0$ at the emission peak of TC1 and TC8, in the eastern dust lane. The SEST spectra of TC8 are corrected for the main beam dilution effect. The IRAM spectra of TC1 are expressed in units of mainbeam brightness temperature.

opacity law $\tau_{v} \propto v^{1.3}$, absorbed by a cold dust layer with an extinction $A_{\mathrm{v}}=30$, which corresponds to a gas column density $N\left(\mathrm{H}_{2}\right)=1.6 \times 10^{22} \mathrm{~cm}^{-2}$, adopting the reddening law derived by Lynds et al. (1985) in the Trifid (Fig. 13). The uncertainties in such a fit are rather large due to the narrow wavelength range observed. We found that introducing an additional component tracing photospheric emission at about $5000 \mathrm{~K}$ provides a better fit to the spectrum. Assuming sizes of $1 \mathrm{AU}$ for the photospheric

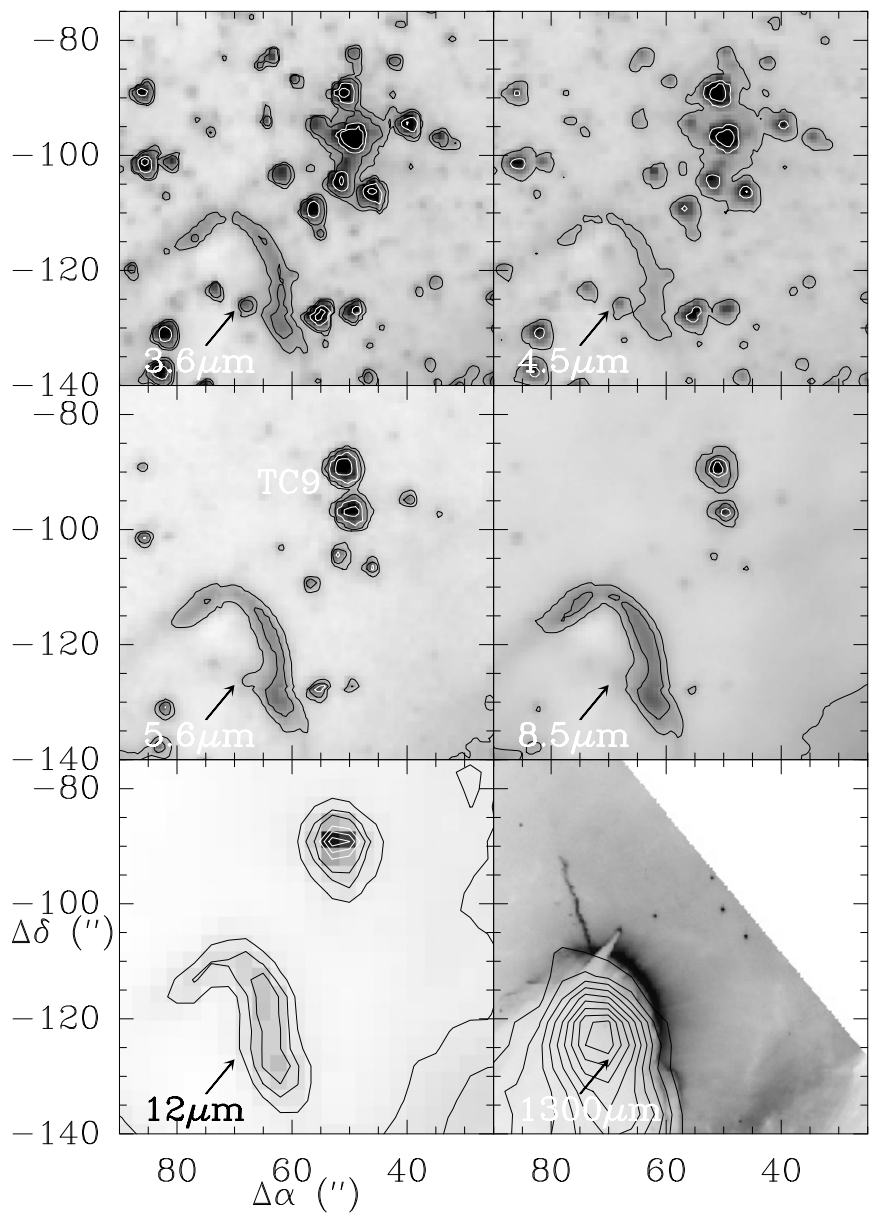

Fig. 12. The TC 2 region seen in the mid-IR with ISOCAM $(8-15 \mu \mathrm{m})$ and SPITZER $(3.6 \mu \mathrm{m}, 4.5 \mu \mathrm{m}, 5.8 \mu \mathrm{m}, 8.0 \mu \mathrm{m})$. In the bottom right panel the $1.3 \mathrm{~mm}$ thermal dust emission (black contours) superposed on a greyscale image of the ionized gas emission as seen in the optical with HST. Contours of cold dust emission are 10, 20, 40, 60, .., $160 \mathrm{mJy} / 11^{\prime \prime}$. The arrow points to the position of the protostellar source.

emission region and of $100 \mathrm{AU}$ for the warm dust component, we estimate a typical gas column density of $3.2 \times 10^{20} \mathrm{~cm}^{-2}$ in the warm dust region. The assumed sizes are arbitrary, as the only constraint we have is that they are less than $1^{\prime \prime}$, the size of the IRAC PSF at $3.6 \mu \mathrm{m}$. The ISOCAM spectrum is typical of young stellar objects in the Class II phase and consistent with the emission arising from a young stellar object surrounded by a protoplanetary disk, embedded in a small gas condensation. The evolutionary age of Class II objects is typically several $10^{5} \mathrm{yr}$ up to a few Myr, i.e. it compares well with the age of the HII region. It suggests that such sources could have formed in the same burst of star formation as the exciting star of M20. From the gas column density of absorbing material, we estimate a mass of 4-8 $M_{\odot}$ for the TC9 core.

Searching for molecular gas emission from TC9, we have detected a clump of gas in the millimeter lines of $\mathrm{CO}$ and its isotopologues (see Fig. 14). The lines peak at $16.5 \mathrm{~km} \mathrm{~s}^{-1}$ (Fig. 15), which implies that TC9 is located on the rear side of the nebula. The clump is hardly resolved at $1.3 \mathrm{~mm}$ in the ${ }^{12} \mathrm{CO} J=2 \rightarrow 1$ transition. The kinetic temperature of the molecular gas is difficult to estimate as the condensation is unresolved by the telescope beam. The ${ }^{12} \mathrm{CO} J=1 \rightarrow 0$ line consists of a narrow line of $7 \mathrm{~K}$ superposed on a broad pedestal of $4 \mathrm{~K}$. Taking into 


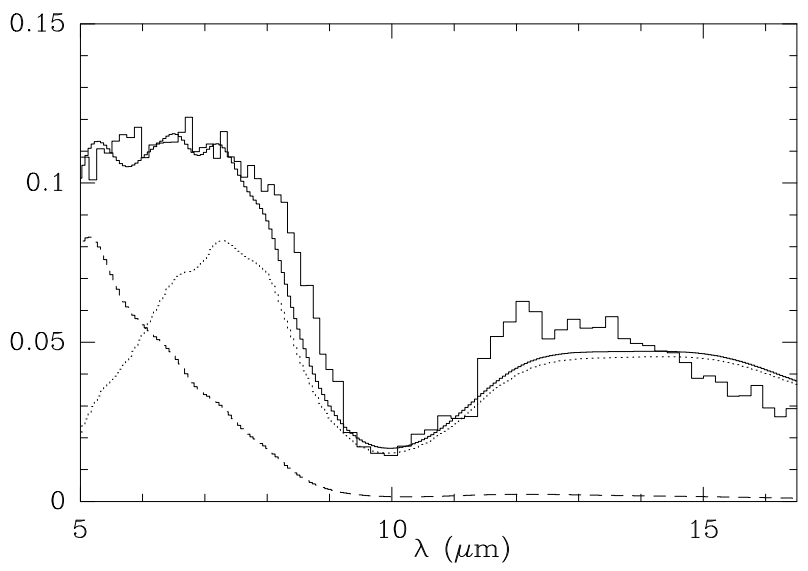

Fig. 13. Spectrum in the range 5-17 $\mu \mathrm{m}$ of TC9, after subtracting the emission of a reference position. The spectrum is fitted by two modified blackbodies with a dust opacity $\tau_{v} \propto v^{1.3}$, at temperatures of $5000 \mathrm{~K}$ (dashed) and $320 \mathrm{~K}$ (dotted) respectively. The sum of the two components is displayed in a thick line.

account the beam dilution effect on the core, we find that the ${ }^{12} \mathrm{CO} J=1 \rightarrow 0$ line brightness has to be less than $35 \mathrm{~K}$.

\section{Discussion}

\subsection{Core properties}

In many cores, self-gravity is comparable to the kinetic energy available, hence plays an important dynamical role in the evolution of the cores. This is the case of cometary globules TC1, TC2, TC8, embedded cores like TC3A, TC4A, TC5, all of them are star-forming condensations. However, the non star-forming condensations TCOA, TC00 are also found in this class. This suggests they might be on the verge of gravitational collapse.

In most of the pre-stellar condensations (e.g. TCOB, 0C, 4C, $6 \mathrm{~A}-\mathrm{D}, 7)$, self-gravity is much lower than the available kinetic energy. This also applies to the evolved cometary globules TC12, $13,14,15,16$ which have already formed protostars, detected with SPITZER. In such objects, the dense gas emission is restricted to the region right behind the bright rim and we do not find evidence of a dense protostellar envelope which would be associated with the SPITZER sources, like in e.g. the young Class0/I TC2-3. Most likely, the bulk of accretion is over in these cometary globules, as only low column densities of material are still available there.

Overall, the star-forming cores detected in our survey have masses of $10 M_{\odot}$ or more (up to 100-200 $M_{\odot}$; see Table 1 ). The two cometary globules which have been studied in detail, TC1 and TC2, exhibit very similar physical properties, in terms of core mass, density, temperature and turbulence. With an average core density of $1.8 \times 10^{5} \mathrm{~cm}^{-3}$ and sound speed $\sigma=0.42 \mathrm{~km} \mathrm{~s}^{-1}$, the Jeans length is $\lambda_{\mathrm{J}}$ is $\approx 0.17 \mathrm{pc}$ in TC1 and TC2, hence approximately the core diameter. It is therefore no wonder that only one protostar was detected in the condensations. In other cases, the Jeans length $\lambda_{\mathrm{J}}$ is less than one core radius and the Jeans mass is a small fraction of the total mass available in the condensation. Hence, the cores appear to be dense and massive enough to give birth to a few stellar objects. This is in agreement with the IRAC maps of TC3, TC4, and TC5 which unveil clusters of protostars.

On the contrary, most of the non star-forming (pre-stellar) condensations have masses of a few $M_{\odot}$, and self-gravity is not large enough to counterbalance the kinetic energy of the gas. The

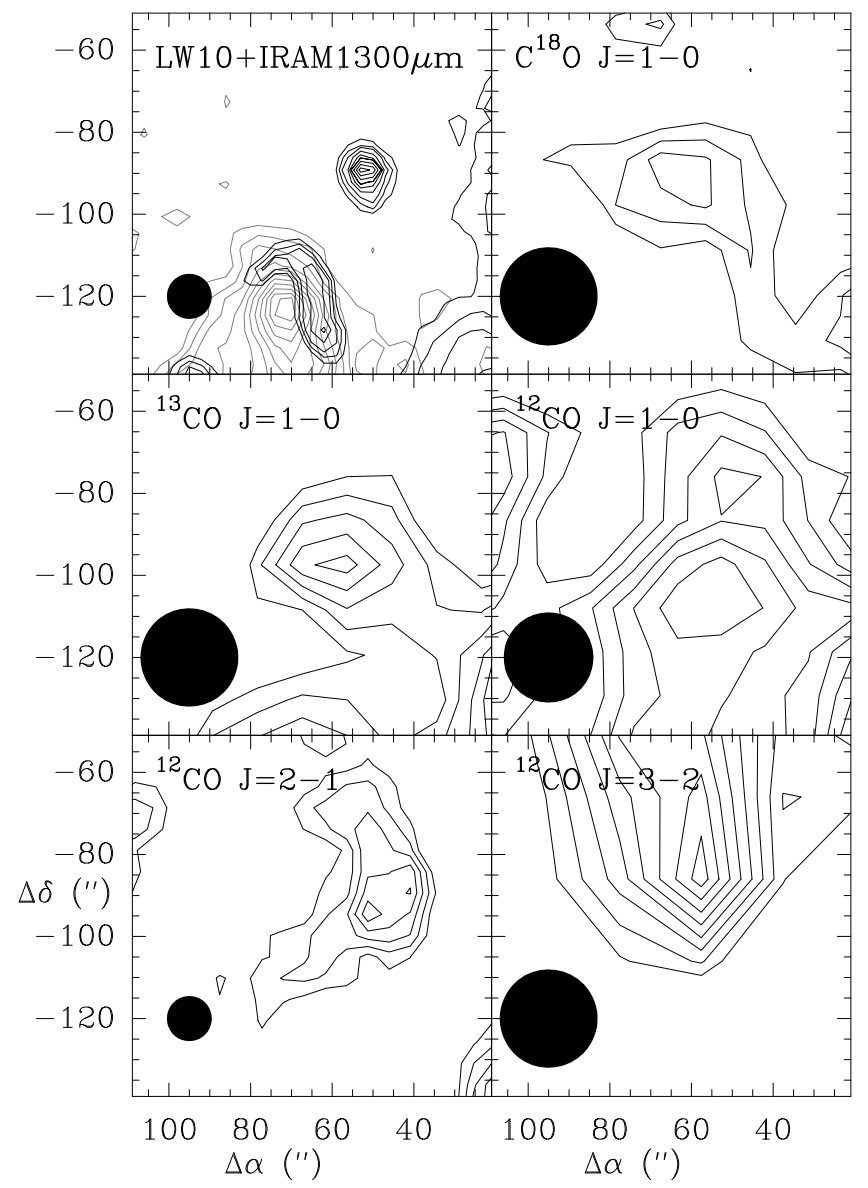

Fig. 14. Mid-infrared (ISOCAM) and millimeter line emission towards TC9. The molecular line flux is integrated between 15.25 and $17.25 \mathrm{~km} \mathrm{~s}^{-1}$. Solid circles indicate the HPBW beam size. Upper left: the mid-infrared $8-15 \mu \mathrm{m}$ is shown in black contours. Levels range from 160 to 180 by 10, 200 to 240 by 20, 240 to 360 by $40 \mathrm{MJy} / \mathrm{sr}$. In thin contours is drawn the $1.3 \mathrm{~mm}$ emission; contours range from 10 to 30 by 10 and from 40 to 150 by $15 \mathrm{mJy} / 11^{\prime \prime}$ beam. We indicate for each panel, the observed transition, the first contour and the contour interval, respectively. Upper right: 0.2 and $0.1 \mathrm{~K} \mathrm{~km} \mathrm{~s}^{-1}$. Middle left: 0.4 and $0.2 \mathrm{~K} \mathrm{~km} \mathrm{~s}^{-1}$. Middle right: 1.6 and $0.2 \mathrm{~K} \mathrm{~km} \mathrm{~s}^{-1}$. Bottom left: 15 and $1 \mathrm{~K} \mathrm{~km} \mathrm{~s}^{-1}$. Bottom right: 14.5 and $0.5 \mathrm{~K} \mathrm{~km} \mathrm{~s}^{-1}$.

only exceptions are TC0A and TC00 $\left(49 M_{\odot}\right)$. We conclude that the cores in $\mathrm{TC} 0$ and $\mathrm{TC} 00$ are privileged sites for the formation of the next generation of stars. We speculate that the gravitational collapse will start as soon as the cores will be compressed by the shock preceding the ionization front. The prestellar cores in TC0 and exhibit physical properties (mass, temperature, density) similar to those of the star-forming cores TC1 and TC2. Hence, we expect that most of the new forming stars will be low- (and/or intermediate) mass stars and will form inside these fragments.

\subsection{Evolutionary sequence for the cores}

The cold dust cores detected in our survey are submitted to a large variety of physical conditions depending on the photoionization in the parent cloud: very deeply embedded cores in massive molecular gas fragments (e.g. TC3-4-5); cometary globules, i.e. cores surrounded by a dense envelope exposed to the ionizing nebular radiation (e.g. TC1-2); cores in an advanced stage of 


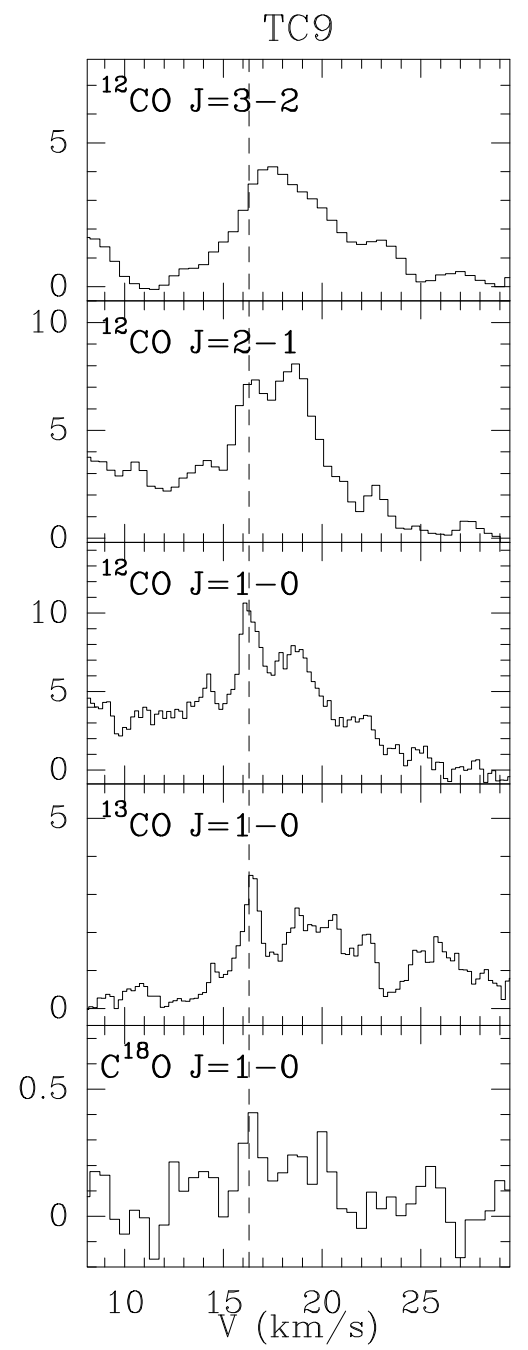

Fig. 15. Molecular line emission observed towards TC9. Fluxes are expressed in units of antenna temperature. The dashed line draws the velocity of the main body gas of TC9.

photoionization, which led them to get rid of their envelope (e.g. TC12-13).

Undoubtedly, such sources are in a very advanced stage of protostellar evolution and photoionization. Some other sources, such as TC9 and TC13, are associated with molecular material detected in line emission, but remain below the detection limit of our millimeter continuum survey.

Our survey allows us to draw the full evolutionary sequence of cometary globules exposed to the ionizing nebular radiation, from their initial stage of formation, as illustrated by the prestellar cores embedded in the fragments of dense molecular gas TC 0 and TC00, to the late stages when the bulk of the envelope has been photoevaporated, like in TC9. Condensations TC1-28 , directly exposed to the harsch nebular ionizing radiation, are still emerging from the low-density layers of the parental molecular cloud. This differs from the situation in M16, another wellstudied HII region, where the erosion of molecular gas condensations by the ionizing radiation has shaped "pillows" in the parent cloud.

The free-free emission was observed towards TC1 at the VLA by Yusef-Zadeh et al. (2005), who derived an electron density of $\approx 2000 \mathrm{~cm}^{-3}$ in the bright rim, i.e. similar to the value measured in the bright rim of TC2. Taking into account that the other properties are similar, the mass-loss rate induced by photoevaporation should be close to the value derived at the surface of TC2: $34 M_{\odot} \mathrm{Myr}^{-1}$. The lifetime of TC1 is therefore $\approx 2 \mathrm{Myr}$, similar to the expected lifetime of an O 7.5 III star (Martins et al. 2005). The photoevaporation timescale of the condensations TC1 and TC2 is long enough to allow the central protostellar objects to accrete the bulk of material from their envelope before being exposed to the ionizing radiation. Hence, the incubators located in the densest part of the molecular cloud, in the dust lanes of the Trifid, will eventually allow their young stellar objects to reach "maturity" (the protoplanetary phase) before being exposed to the harsh ionizing radiation.

The fate of TC9, which is located in a less dense region on the rear face of the nebula, is somewhat different. TC9 consists of a small core of dense gas and dust. The central source is surrounded by sufficiently large amount of materials that the $9.7 \mu \mathrm{m}$ silicate band is still detected in absorption. Besides, the SED of the central source is typical of Class II objects, consistent with the active accretion phase being now terminated. We adopt as a rough estimate of the ionizing flux impinging on TC9 the one measured at the surface of TC2: $\Phi \sim 10^{10} \mathrm{~cm}^{-2} \mathrm{~s}^{-1}$. This is justified because TC 2 and TC9 have similar projected distances to the exciting star of the nebula. Using Eq. (36) of Lefloch \& Lazareff (1994), which relates the mass-loss rate to the size of the condensation and the ionizing photon flux, we find a mass loss rate of $\simeq 8 M_{\odot} \mathrm{yr}^{-1}$, where we have adopted a mean radius of $\simeq 8^{\prime \prime}$; hence, the condensation will have been photoevaporated in a few $10^{5} \mathrm{yr}$, while the young stellar objects TC1 and TC2 will still be shielded from the ionizing radiation. TC9 is a clear case of a protostellar core overrun by the ionization front, which is about to disappear in the nebular gas.

IRS 6-7, TC11-12,13 illustrate the ultimate stages of early stellar evolution when the parental envelope of the newly born stars has been almost fully photoevaporated and hardly any cold molecular gas emission is detected. In these objects, self-gravity fails to bind the structure, as evidenced by the ratio between the virial and the actual mass of the condensations. We note however that the gravitational potential due to the central object(s) was ignored, so that the actual discrepancy might be less.

\subsection{Core formation and filaments}

The overall distribution of molecular material differs a lot from the free-free emission distribution observed with the VLA, which traces an almost perfect Stromgren sphere (CL98). Most of the protostellar cores are encountered in filamentary structures. Some of them, like TC1, TC2, TC4, are detected in the large-scale photon-dominated region but its not the case for all of them. In particular, cores and fragments in WF, such as TC00, 6, 7 are encountered far away from the HII region.

Since the WF filament stretches over $\approx 10 \mathrm{pc}$, far beyond the ionization radius of M 20 and connects several complexes together, we can exclude that it formed from the fragmentation of the layer of molecular material swept-up in the expansion of the HII region, as in the "collect and collapse" scenario (Elmegreen \& Lada 1977; Elmegreen 1989). WF was already present before the ignition of the HII region, and so were probably the dust lanes of the Trifid, and other filaments, like in the TC4 region. The separation of the massive condensations in WF along the filament is approximately constant, equal to $1.1 \times 10^{19} \mathrm{~cm}^{-2}(3.6 \mathrm{pc})$; this provides additional evidence for the fragmentation scenario.

In the filaments EL, SL, and TC4f, the size of the cores is very close to the diameter of these filaments, and their Jeans length. This fact supports the idea that the cores form as a consequence of the fragmentation of the filaments. Also, many of 
the pre-stellar cores, such as TC6, 10, 11 are not gravitationallybound, which implies that they probably formed dynamically inside the filaments.

We explore here the scenario that all the condensations detected in our survey actually result from the fragmentation of filamentary clouds. These questions will be addressed more thoroughly in a detailed analysis of the molecular gas dynamics in a forthcoming article (Lefloch 2008, in prep.).

\subsection{Fragmentation of WF}

The map of the thermal dust emission shows that the material of WF is concentrated mainly in the massive condensations TC00, TC0, TC3, TC5. From the average gas velocity dispersion in the TC0-TC3-TC6 region $\left(\sigma=0.7 \mathrm{~km} \mathrm{~s}^{-1}\right)$, we estimate the virial mass per unit length $m_{\mathrm{v}}=2 \sigma^{2} / G=220 M_{\odot} \mathrm{pc}^{-1}$, which compares well with the mass per unit length in WF (Sect. 4). We conclude that the filament was initially self-gravitating. Under the simplifying hypothesis that WF was initially in hydrodynamical equilibrium, and that it fragmented under some external perturbations, the properties of the fragments will be mainly determined by the fastest growing mode of the perturbation.

We present here a simple analysis, based on the results of the continuum survey. The characteristic size for a filament in hydrodynamical equilibrium is given by:

$$
H=0.0035\left(\frac{c_{\mathrm{s}}}{0.3 \mathrm{~km} \mathrm{~s}^{-1}}\right)\left(\frac{n_{0}}{2 \times 10^{6} \mathrm{~cm}^{-3}}\right)^{-1 / 2} \mathrm{pc}
$$

where $c_{\mathrm{s}}$ is the effective sound speed (=1 d velocity dispersion $\sigma$ ) and $n_{0}$ is the gas density in the central region of the filament (see Nakamura et al. 1993), and is approximately twice the average density of the filament. Hence, we find $H=0.18 \mathrm{pc}$ and an effective filament radius of $R_{\mathrm{e}}=2 \sqrt{2} H=0.51 \mathrm{pc}$. The actual diameter of the filament is not so easy to measure from the emission maps; the initial diameter of the filament could have been larger before undergoing external compression. The observations at SEST and CSO show a somewhat larger radius (of about $0.6-0.8 \mathrm{pc}$ ), though not inconsistent with these results. For a purely hydrodynamically supported filament, the most unstable mode of axisymmetric perturbations has a wavelength $\lambda=22.0 H \simeq 4.0 \mathrm{pc}$, a value in reasonable agreement with the mean separation between fragments $\approx 400^{\prime \prime}=3.6 \mathrm{pc}$.

A better agreement is obtained under the assumption that the filament is threaded by a magnetic field and that equipartition is reached between kinetic and magnetic energies. The role of the magnetic field must be taken into account as it provides additional support against the filament self-gravity; as a consequence the filament increases its scale height and its effective radius $R_{\mathrm{e}}$. With the same physical parameters and assuming equipartition between magnetic and kinetic energy, the scale height $H^{\prime}$ becomes $H^{\prime}=H \times \sqrt{2}=0.26 \mathrm{pc}$ and the equivalent radius $R_{\mathrm{e}}=0.73 \mathrm{pc}\left(90^{\prime \prime}\right)$. The wavelength of the most unstable mode for axisymmetric perturbations in a magnetized filament is then $\lambda=13.0 H^{\prime}=3.4 \mathrm{pc}$. Overall, these results agree well with the observations, in particular the constraint on the diameter of the filament is better fulfilled.

Inspection of the fragment along WF shows a marked trend. Whereas TC3 and TC5 fragments have very comparable masses $\left(\approx 800 M_{\odot}\right)$, the mass of each newly encountered fragment when moving North along the filament decreases, by a factor about 2 (see Table 2). The material distribution of TCO0 seems less structured than in TC0, itself less structured than in TC3 and TC5.
This suggests that the fragmentation of the filament could have started first with the formation of TC3 and TC5, then proceeded to the North of the filament, where TC0 and TC00 would represent subsequent generations of fragments.

The sequential star formation triggered by the expansion of an HII region, impacting a filamentary cloud has been studied numerically by Fukuda \& Hanawa (2000). Their simulations show that under a wide range of physical conditions it takes about 10 times the radial sound crossing time

$$
\begin{aligned}
\tau & =H / c_{\mathrm{s}} \\
& =1.7 \times 10^{4}\left(\frac{n_{0}}{2 \times 10^{6} \mathrm{~cm}^{-3}}\right)^{-1 / 2} \mathrm{yr}
\end{aligned}
$$

to form the first condensations in the shocked filament. In the case of WF, this time would be as large as $4 \mathrm{Myr}$, almost one order of magnitude larger than the age estimated for the Trifid (L02). Despite all the uncertainties in the age determinations, the detection of photoionized protoplanetary disks in the central cluster of the HII region (L01) implies that the nebula is young. It is more likely that fragmentation in WF was initiated by some other events than the interaction with M20. An interesting result of these simulations is that fragmentation can occur at much earlier times as more kinetic energy is injected in the shock. An alternate scenario is that fragmentation was already ongoing before the ignition of the nebula.

A large number of observations support the existence of a physical interaction between the young W28 SNR and the surrounding molecular gas (Wootten 1981; Frail \& Mitchell 1998; Arikawa et al. 1999; Rho \& Borkowski 2002; Yusef-Zadeh et al. 2000; Reach et al.). It is not clear however if such events as energetic as supernova remnants could trigger the fragmentation of the filament on such short timescales. On the other hand, the characteristic timescales of TC3 and TC5 are fully compatible with a dynamical interaction between WF and W28. As discussed above, the free-fall gravitational timescale in the cores of TC3 and TC5 are extremely short, of the order $10^{4} \mathrm{yr}$, and similar to the age of the protostellar sources in TC3 and TC5. This is in excellent agreement with the estimated age of the W28 supernova remnant $\left(3.3 \times 10^{4} \mathrm{yr}\right.$, Velázquez et al. 2002).

In order to confirm this hypothesis, it should be investigated whether the impact of the SNR shock on the WF filament, the shock could trigger the gravitational collapse of the core and the formation of the protostellar cluster. The location of TC5 at the edge of the S28 shell star formation strongly suggests that star formation has indeed been triggered in the core as soon as the supernova shock hit the condensation.

\subsection{Fragmentation of TC4f}

In TC4f filament, the different cores $(4 \mathrm{a}, 4 \mathrm{~b}, 4 \mathrm{c}, 4 \mathrm{~d})$ are located along the filament, at the border of the nebula. A detailed analysis of the physical conditions showed evidence for compression of the filament gas (LCO0). No evidence of velocity gradient was found between the various molecular gas tracers. If the collapse of the cores had started before compression of the surface layers occurred, lines with different optical depths should probe different regions of the core, hence tracing regions at different velocity. There is no such velocity gradient observed, between the optically thick CS and the optically thin $\mathrm{C}^{18} \mathrm{O}$ line. Most likely, the cores TC4a-b-c-d formed after compression of the filament. The properties of the cores are consistent with them being fragments of this layer (LC00). The timescale for gravitational collapse of a decelerating shocked gas layer is $\tau \sim 0.25\left(G \rho_{0}\right)^{-1 / 2}$, 


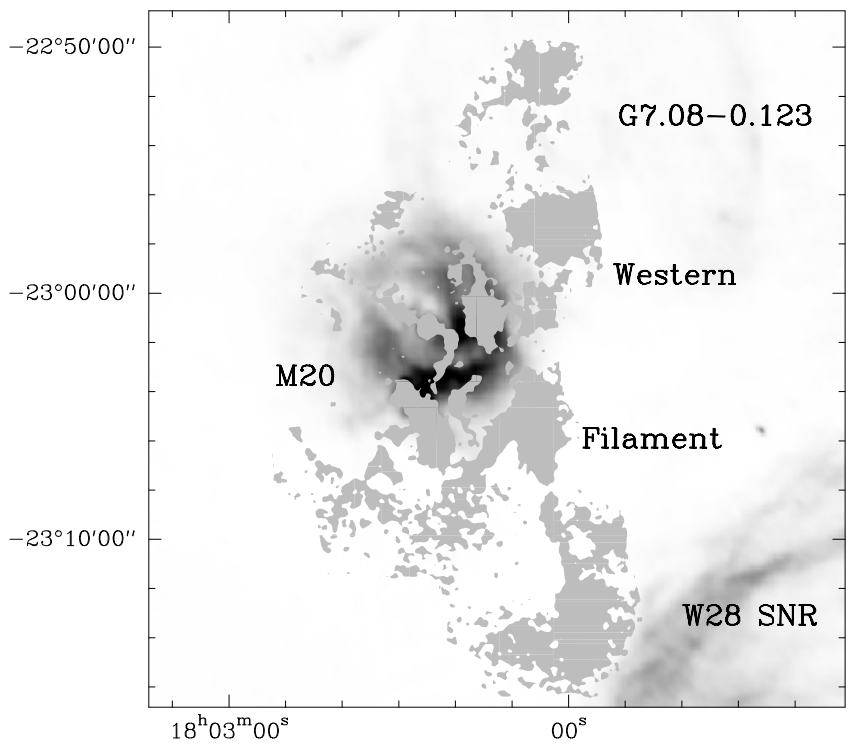

Fig. 16. Map of the $1300 \mu \mathrm{m}$ thermal dust emission superposed on a map of the $20 \mathrm{~cm}$ free-free emission of the region in greyscale (from Yusef-Zadeh et al. 2000).

where $\rho_{0}$ is the typical (pre-shock) density of the parent molecular cloud (Elmegreen 1989). The average density determined over WF $\left(2000 \mathrm{~cm}^{-3}\right)$ provides a good estimate of $\rho_{0}$. The typical timescale for the fragmentation of TC4f density is therefore $0.35 \mathrm{Myr}$, compatible with the age estimated for M 20. To summarize, there is good observational evidence that TC4f is a filament compressed by the expansion of the HII region, which subsequently became gravitationally unstable and formed young stellar objects.

\subsection{Fragmentation in the dust lanes}

We have carried out a similar analysis for filaments SL and EL. The results are summarized in Table 3. Unlike WF and TC4f, the southern and the eastern lanes are only weakly gravitationally bound. They are probably confined by the external pressure of the ambient medium or by a helicoidal and/or toroidal magnetic field (see Fiege \& Pudritz 2000a). This is consistent with the less efficient star-forming efficiency observed there. However, the actual mass per unit length of the lanes is less since the O star turned on and has started to photoevaporate the surrounding molecular gas. It is not possible to accurately estimate the mass loss rate of both filaments as the orientation of the dust lanes makes it difficult to discriminate the contributions of the photoionized lane surface layers and the nebular gas to the freefree emission; moreover we do not know the exact distance of the $\mathrm{O}$ star to the lanes. A reasonable order of magnitude can be obtained by adopting the expression of the mass-loss rate derived for cometary globules to the filaments, and assuming an impinging ionizing photon flux similar to the one measured towards TC2. Under such hypothesis, we estimate a typical mass loss rate of $85 M_{\odot} \mathrm{Myr}^{-1}$ for a filament $1 \mathrm{pc}$ long. With an estimated age of $0.4 \mathrm{Myr}$, it means that SL has evaporated about half its initial mass, which was $\approx 85 M_{\odot} \mathrm{pc}^{-1}$. Similarly EL has lost about $1 / 3$ of its initial mass. In both cases, the initial mass of the filament was noticeably closer to the virial mass; hence, gravity was playing a more important role in the overall mechanical equilibrium of the clouds, which means that the discrepancy between filament and cores with respect to self gravity was probably much less.

Fiege \& Pudritz (2000a,b) have investigated the stability and fragmentation of filamentary clouds truncated by an external pressure and threaded by a helical magnetic field. They addressed the stability of the filaments to gravitational fragmentation and axisymmetric MHD driven instabilities. The authors have identified two well separated types of unstable modes, depending on the toroidal to poloidal magnetic flux ratio $\Gamma_{\Phi} / \Gamma_{z}$, which join together when $\Gamma_{\Phi} / \Gamma_{z}=2$. At low values, the instability mode is gravity-driven and for pressure truncated filaments such as EL and SL, the growth timescale is $\sim 1 \mathrm{Myr}$ or longer. It implies that fragmentation would have started well before the birth of the exciting star of the nebula. The unstable MHD-driven instabilities triggered above $\Gamma_{\Phi} / \Gamma_{z}=2$ can have very large growth rates compared with gravity-driven modes, and hence much shorter fragmentation timescales.

The regime of instability relevant to the lanes can be determined from the ratio of the fragment separation $\lambda$ to the filament diameter $D$. In our case, one has $\lambda / D=1.7$ for EL and 1.5 for SL, respectively. We note however that the photoevaporation of the outer, low-density, layers of the filaments underestimate the actual ratio, before ionization turns on. The radius of the filaments in the neutral parental cloud, was definitely larger, so that the ratio is probably closer to 1 or even less. From Fig. 17 of $\mathrm{FPb}$ it comes that $\Gamma_{\Phi} / \Gamma_{z}=2-3$, i.e. MHD driven instabilities can propagate in the two filaments. The most unstable modes are characterized by a growth rate $\omega^{2} \approx(0.003-0.1) \times\left(4 \pi G \rho_{0}\right)^{-1}$. The growth timescale of these modes $\left(\omega^{-1}\right)$ can be as low as $4 \times 10^{5} \mathrm{yr}$ in the case $\left(\Gamma_{\Phi} / \Gamma_{z} \simeq 3\right)$. This is of the same order as the radial sound crossing time $R_{\mathrm{e}} / c_{\mathrm{s}}=0.3 \mathrm{Myr}$, a constraint to be met for an instability to develop along the filament without destroying the entire structure. We conclude that axisymmetric MHD-driven instabilities can in principle account for the fragmentation observed in the dust lanes of the nebula, on a timescale compatible with its age. However it puts some constraints on the magnetic field structure of the filaments.

At this stage, our work suggests that the magnetic field probably plays an effective role in the dynamics of the filaments of the Trifid and in their fragmentation. Whatever the relative importance of hydrodynamical and magnetic support, the main conclusion is that the various condensations TC00, TC0, TC3, TC5 most likely formed from the fragmentation of WF. Both fast and slow fragmentation processes are at work in the Trifid, and depend on the initial conditions in the environment of the nebula. Zeeman and polarimetric measurements of the magnetic field are needed to quantify its role in the support against gravity and the formation process(es) of the stellar incubators.

\section{Conclusions}

Our mapping of the Trifid Nebula at millimeter, submillimeter and mid-infrared wavelengths has allowed us to characterize the gas and dust distribution. Thirty three cores have been identified, which cover all the stages of protostellar evolution, from the early prestellar to the late protoplanetary phases. Sixteen cores are found to display evidence of star formation.

The cores are found to lie at various stages of photoionization, from the deeply embedded molecular phase to the cometary phase and the late stages of photoevaporation. Taking into account also the previously studied objects like TC2 (LC02), all cometary globules display very similar properties. They have typical sizes of 0.1 to $0.2 \mathrm{pc}, \mathrm{H}_{2}$ densities ranging from $\approx 10^{5} \mathrm{~cm}^{-3}$ to $10^{6} \mathrm{~cm}^{-3}$, and masses of a few $M_{\odot}$ up to a few 
tens $M_{\odot}$. They appear to form at most one intermediate-mass object. Hence, we conclude that photoionization does not increase the star formation efficiency in the individual cores. We find evidence of clustering only in the shocked, dense condensations, such as TC3 and TC4 (previously studied in LC00) and TC5.

Comparison of the line and continuum emission properties shows hardly any evidence for molecular depletion inside the condensations. Only moderate molecular depletion has been reported towards the densest sources (TC3; LC00). The midinfrared 5-17 $\mu \mathrm{m}$ flux detected with ISOCAM towards TC1 and TC9 is consistent with the emission of a hot central source, with dimensions typical of protoplanetary disks, which is absorbed by the parental envelope of cold dust and gas. ISOCAM spectroscopy has revealed the presence of large amounts of $\mathrm{CO}_{2}$ ices in cometary globule $\mathrm{TC} 1$, thereby unveiling the potentially rich composition of icy grain mantles in protostellar environments in the Trifid. As the ice composition reflects the various processes that take place on grains and the chemical evolution of the protostellar envelope, mid-infrared observations should allow us to investigate how the chemical composition of the circumstellar envelopes varies along with protostellar evolution in the HII region.

The cloud material is distributed in filamentary structures of relatively high density (a few $10^{3} \mathrm{~cm}^{-3}$ ) and gas column density $N(\mathrm{H})$ of a few $10^{22} \mathrm{~cm}^{-2}$. Their properties compare well with those of IRDCs and the Orion filaments reported by Maddalena et al. (1986). The length of these structures ranges from about $1 \mathrm{pc}$ to $15 \mathrm{pc}$ or more. Since some of them extend far beyond the ionization front of the HII region, these filamentary structures cannot have formed in a "collect and collapse" manner. They were pre-existing the birth of M 20 and reflect the initial conditions of the parent molecular cloud.

Among the four filaments studied in greater detail by us, two of them are confined by self-gravity (WF and TC4f), whereas the other two are presently not. Taking into account the photoevaporation of the surface layers of the latter, they were probably self-gravitating too before the onset of photoionization. All these filaments are fragmented in condensations with typical masses of a few hundreds of $M_{\odot}$. The fragmentation of the filaments can be accounted for by MHD-driven instabilities, although the exact importance of the magnetic field in the region remains to be quantified. Comparison with numerical simulations shows that the impact of M20 on the filaments is too recent to have triggered their fragmentation, with the exception of the TC4f filament. It may well be, however, that this fragmentation occurred at an earlier stage or was accelerated under the influence of high energy shock interactions with young supernova remnants. It is not clear if the mass distribution of the fragments in the filament WF results from the propagation of instabilities inside the filament, hence triggering the formation of the subsequent generations of cores. Conversely, the small-scale fragmentation reported in the filament TC4f provides a convincing example of a filament compressed and destabilized in the expansion of the HII region, well accounted for by the modelling of Elmegreen (1989). The mass of the individual fragments formed in the TC4f filament is rather low $\left(\sim 10 M_{\odot}\right)$, whereas the mass of the objects in WF is typically ten times higher.

The star formation going on in the dense cores TC3 and TC5 is characterized by very short dynamical timescales (about $10^{4} \mathrm{yr}$ ), comparable to the age of the nearby W28 supernova remnant. TC5 is a good candidate to investigate the possibility of SNR shocks as a trigger of star formation. Therefore, the environmental conditions appear to have played a very important role in determining the star forming conditions in the
Trifid Nebula. The kinematics of the gas will be investigated in a forthcoming paper, in order to search for signatures of these environmental conditions and of possible interactions with the highly energetic phenomena which have recently taken place in the neighbourhood.

Acknowledgements. We thank the referee for his many detailed comments, which greatly improved the reading of the manuscript. J. R. Pardo and J. Cernicharo acknowledge Spanish Ministerio de Educacion y Ciencia for supporting this research under grants AYA2003-2785, AYA2006-14876, ESP200400665, ESP2007-65812-C02-01, Communidad de Madrid for research grant ASTROCAM S-0505ESP-0237, and the Molecular Universe FP6 Marie Curie Research Training Network.

\section{References}

André, P., Ward-Thompson, D., \& Barsony, M. 2000, in Protostar \& Planets IV Bacmann, A., Lefloch, B., Ceccarelli, C., et al. 2002, A\&A, 389, L6

Bertoldi, F. 1989, ApJ, 346, 735

Beuther, H., Churchwell, E., McKee, C. F., \& Tan, J. C. 2007, in Protostars and Planets V, ed. B. Reipurth, D. Jewitt, \& K. Keil (Tucson: Univ. Arizona Press) Broguiere, D., Neri, R., \& Sievers, A. 1995, NIC bolometer users guide (IRAM internal report)

Cernicharo, J., Lefloch, B., Cox, P., et al. 1998, Science, 282, 462 (CL98)

Cernicharo, J., Noriega-Crespo, A., Cesarsky, D., et al. 2000, Science, 288, 649 Deharveng, L., Lefloch, B., Zavagno, A., et al. 2003, A\&A, 408, L25 Deharveng, L., Lefloch, B., Massi, F., et al. 2006, A\&A, 458, 191 Dobashi, K., Yonehura, Y., Matsumoto, T., et al. 2001, PASJ, 53, 85 Elmegreen, B. G. 1989, ApJ, 340, 786

Elmegreen, B. G. 2002, ApJ, 577, 206

Elmegreen, B. G., \& Lada, C. J. 1977, ApJ, 214, 725

Fiege, J. D., \& Pudritz, R. E. 2000a, MNRAS, 311, 85

Fiege, J. D., \& Pudritz, R. E. 2000b, MNRAS, 311, 105

Fukuda, N., \& Hanawa, T. 2000, ApJ, 533, 911

Healy, K. R., Hester, J. M., \& Claussen, M. J. 2004, ApJ, 610, 835

Hester, J. J., Scowen, P. A., \& Sankrit, R. 1996, AJ, 111, 2349

Lefloch, B., \& Lazareff, B. 1994, A\&A, 289, 559 (LL94)

Lefloch, B., \& Lazareff, B. 1995, A\&A, 301, 522

Lefloch, B., \& Cernicharo, J. 2000, ApJ, 545, 340 (LC00)

Lefloch, B., \& Cernicharo, J. 2003, in The Young Local Universe, Proc. of the XXXIXth Rencontres de Moriond, ed. A. Chalabaev, T. Montmerle, \& J. Tran Thanh Van, 353

Lefloch, B., Lazareff, B., \& Castets, A. 1997, A\&A, 324, 249

Lefloch, B., Cernicharo, J., Cesarsky, D., et al. 2001, A\&A, 368, L13 (L01)

Lefloch, B., Cernicharo, J., Rodriguez, L. F., et al. 2002, ApJ, 581, 335 (L02)

Lynds, B., \& O’Neil, E. J. Jr. 1986, AJ, 92, 1125

Lynds, B., Canzian, B. J., \& O’Neil, E. J. Jr. 1985, ApJ, 288, 164

Martens, F., Schaerer, D., \& Hillier, D. J. 2005, A\&A, 436, 1049

Maddalena, R. J., Morris, M., Moscowitz, J., et al. 1986, ApJ, 303, 375

Minier, V., Burton, M. G., Hill, T., et al. 2005, A\&A, 429, 945

Motte, F., André, P., \& Neri, R. 1998, A\&A, 336, 150

Pestalozzi, M. R., Minier, V., \& Booth, R. S. 2005, A\&A, 432, 737

Nakamura, F., Hanawa, T., \& Nakano, T. 1993, PASJ, 45551

Panagia, N. 1973, AJ, 78, 929

Reipurth, B. 1983, A\&A, 117, 183

Reipurth, B., Bally, B., Fesen, R. A., \& Devine, D. 1998, Nature, 396, 343

Rho, J., \& Borkowski, K. J. 2002, ApJ, 575, 201

Rho, J., Corcoran, M. F., Chu, Y. H., \& Reach, W. T. 2001, ApJ, 562, 446

Rho, J., Reach, W., Lefloch, B., \& Fazio, G. 2006, ApJ, 643, 965

Rosado, M., Esteban, C., Lefloch, B., Cernicharo, J., \& García-López, R. J. 1999, AJ, 118, 2962

Spitzer, L. 1978, Physical Processes in the Interstellar Medium (New-York: Wiley), 107

Sugitani, K., Fukui, Y., \& Ogura, K. 1991, ApJS, 77, 59

Tomisaka, K. 1995, ApJ, 328, 226

Velázquez, P. F., Dubner, G. M., Goss, W. M., \& Green, A. J. 2002, AJ, 124, 2145

Walborn, N. R. 1973, AJ, 78, 1067

Walmsley, C. M., Pineau des Forêts, G., \& Flower, D. R. 1999, A\&A, 342, 542

White, G. J., Nelson, R. P., Holland, W. S., et al. 1999, A\&A, 342, 233

Whitworth, A. P., Bhattal, A. S., \& Chapman, S. J. 1994, MNRAS, 268, 291

Yamaguchi, R., Saito, H., Mizuno, N., et al. 1999, PASJ, 51, 791

Yusef-Zadeh, F., Shure, M., Wardle, M., \& Kassim, N. 2000, ApJ, 540, 842

Yusef-Zadeh, F., Biretta, J., \& Geballe, T. R. 2005, AJ, 130, 1171

Zavagno, A., Pomares, M., Deharveng, L., et al. 2007, A\&A, 472, 835 
B. Lefloch et al.: Star formation in the Trifid Nebula, Online Material $p 1$

Table 5. Line parameters of the various transitions observed (intensity in $\mathrm{K}$, velocity peak and linewidth in $\mathrm{km} \mathrm{s}^{-1}$ ) at the brightness peak of the condensations. The intensity of the $\mathrm{C}^{18} \mathrm{O}$ and $\mathrm{CS}$ lines is expressed in units of main-beam brightness temperature. The symbol "-" implies that the relevant transition has not been observed. NG means that the line profile is too complex to be fit by simple Gauss functions.

\begin{tabular}{|c|c|c|c|c|c|c|c|c|}
\hline Name & Emission Peak & $\begin{array}{c}\mathrm{CO} \\
J=1 \rightarrow 0\end{array}$ & $\begin{array}{c}\mathrm{CO} \\
J=2 \rightarrow 1\end{array}$ & $\begin{array}{c}\mathrm{CO} \\
J=3 \rightarrow 2\end{array}$ & $\begin{array}{c}\mathrm{C}^{18} \mathrm{O} \\
J=1 \rightarrow 0\end{array}$ & $\begin{array}{c}{ }^{13} \mathrm{CO} \\
J=1 \rightarrow 0\end{array}$ & $\begin{array}{c}\text { CS } \\
J=2 \rightarrow 1\end{array}$ & $\begin{array}{c}C S \\
J=3 \rightarrow 2\end{array}$ \\
\hline TCOA & $\left(-238^{\prime \prime},+294^{\prime \prime}\right)$ & $\begin{array}{c}22.0 \\
10.5 \\
3.0\end{array}$ & $\begin{array}{c}23.7 \\
10.2 \\
3.0\end{array}$ & $\begin{array}{c}12.5 \\
10.0 \\
3.0\end{array}$ & $\begin{array}{c}1.7 \\
10.4 \\
1.8\end{array}$ & $\begin{array}{c}12.4 \\
10.3 \\
1.8\end{array}$ & $\begin{array}{c}2.5 \\
10.5 \\
1.8\end{array}$ & $\begin{array}{c}3.1 \\
10.5 \\
1.5\end{array}$ \\
\hline TCOB & $\left(-302^{\prime \prime},+292^{\prime \prime}\right)$ & $\begin{array}{c}22.5 \\
9.9 \\
2.8\end{array}$ & $\begin{array}{c}22.5 \\
10.0 \\
2.8\end{array}$ & $\begin{array}{c}16.4 \\
10.2 \\
3.4\end{array}$ & $\begin{array}{c}1.7 \\
10.6 \\
0.8\end{array}$ & $\begin{array}{c}14.7 \\
10.3 \\
1.5\end{array}$ & $\begin{array}{c}2.30 \\
10.60 \\
1.0\end{array}$ & $\begin{array}{c}2.67 \\
10.55 \\
0.95\end{array}$ \\
\hline TCOC & $\left(-268^{\prime \prime},+275^{\prime \prime}\right)$ & $\begin{array}{c}20 \\
10.9 \\
1.7\end{array}$ & $\begin{array}{c}22.0 \\
10.9 \\
1.7\end{array}$ & $\begin{array}{c}13.8 \\
11 \\
2.0\end{array}$ & $\begin{array}{c}1.5 \\
10.6 \\
1.0\end{array}$ & $\begin{array}{c}11.2 \\
10.5 \\
1.3\end{array}$ & $\begin{array}{c}2.5 \\
10.5 \\
1.2\end{array}$ & $\begin{array}{c}2.75 \\
10.5 \\
1.1\end{array}$ \\
\hline TCOD & $\left(-270^{\prime \prime},+252^{\prime \prime}\right)$ & $\begin{array}{c}20.0 \\
9.8 \\
3.8\end{array}$ & $\begin{array}{c}19.0 \\
9.8 \\
4.0\end{array}$ & $\begin{array}{c}15.7 \\
10.1 \\
3.5\end{array}$ & $\begin{array}{c}1.1 \\
10.2 \\
1.2\end{array}$ & $\begin{array}{l}6.8 \\
9.8 \\
1.8\end{array}$ & $\begin{array}{c}1.2 \\
10.4 \\
1.4\end{array}$ & $\begin{array}{c}1.4 \\
10.6 \\
0.9\end{array}$ \\
\hline $\mathrm{TC} 1$ & $\left(+16^{\prime \prime},+33^{\prime \prime}\right)$ & $\begin{array}{l}19.6 \\
3.2 \\
2.6\end{array}$ & $\begin{array}{c}25.4 \\
3.2 \\
3.2\end{array}$ & $\begin{array}{c}15.9 \\
3.55 \\
3.2\end{array}$ & $\begin{array}{l}1.4 \\
3.7 \\
1.3\end{array}$ & $\begin{array}{l}12 \\
3.8 \\
1.7\end{array}$ & $\begin{array}{c}3.0 \\
3.55 \\
1.0\end{array}$ & $\begin{array}{c}3.0 \\
3.85 \\
1.1\end{array}$ \\
\hline $\mathrm{TC} 2^{1}$ & $\left(+72^{\prime \prime},-120^{\prime \prime}\right)$ & $\begin{array}{c}31.0 \\
7.7 \\
2.2\end{array}$ & $\begin{array}{l}29.7 \\
7.5 \\
2.4\end{array}$ & $\begin{array}{l}18.7 \\
8.2 \\
2.9\end{array}$ & $\begin{array}{l}2.3 \\
7.7 \\
1.0\end{array}$ & $\begin{array}{c}13.0 \\
7.65 \\
1.6\end{array}$ & $\begin{array}{l}3.0 \\
7.7 \\
1.3\end{array}$ & $\begin{array}{l}4.4 \\
7.7 \\
1.3\end{array}$ \\
\hline $\mathrm{TC} 3 \mathrm{~A}^{2}$ & $\left(-246^{\prime \prime},-220^{\prime \prime}\right)$ & NG & NG & NG & $\begin{array}{c}5.1 \\
21.6 \\
1.9\end{array}$ & $\begin{array}{c}8.5 \\
21.9 \\
2.9\end{array}$ & $\begin{array}{c}1.5 \\
22.1 \\
5.8^{\ddagger}\end{array}$ & $\begin{array}{c}2.70 \\
20.7 \\
1.9\end{array}$ \\
\hline $\mathrm{TC}_{4} \mathrm{~A}^{2}$ & $\left(-150^{\prime \prime},-246^{\prime \prime}\right)$ & NG & NG & NG & $\begin{array}{c}4.3 \\
21.3 \\
1.2\end{array}$ & $\begin{array}{c}7.4 \\
22.1 \\
2.3\end{array}$ & $\begin{array}{c}2.1 \\
22.6 \\
2.7\end{array}$ & $\begin{array}{c}2.5 \\
22.5 \\
2.6\end{array}$ \\
\hline $\mathrm{TC}^{3}$ & $\left(-360^{\prime \prime},-640^{\prime \prime}\right)$ & NG & NG & NG & - & - & $\begin{array}{c}0.34^{+} \\
21.1 \\
3.0\end{array}$ & $\begin{array}{c}0.37^{+} \\
21.4 \\
4.6\end{array}$ \\
\hline TC6 & $\left(-280^{\prime \prime},-440^{\prime \prime}\right)$ & NG & NG & NG & _ & $\begin{array}{rl}3.1 & 2.1 \\
18.9 & 16.2 \\
2.8 & 2.5\end{array}$ & $\begin{array}{c}0.740 .65^{\dagger} \\
20.016 .5 \\
3.01 .6\end{array}$ & $\begin{array}{cc}0.49 & 0.35^{\dagger} \\
20.0 & 16.5 \\
2.8 & 1.6\end{array}$ \\
\hline TC8 & $\left(+94^{\prime \prime},+87^{\prime \prime}\right)$ & $\begin{array}{c}21.4 \\
3.8 \\
2.7\end{array}$ & $\begin{array}{c}21.4 \\
3.6 \\
3.0\end{array}$ & $\begin{array}{c}15.7 \\
3.85 \\
3.0\end{array}$ & $\begin{array}{l}1.2 \\
4.1 \\
1.1\end{array}$ & $\begin{array}{l}9.6 \\
3.7 \\
1.7\end{array}$ & $\begin{array}{l}1.9 \\
3.5 \\
1.7\end{array}$ & $\begin{array}{l}2.2 \\
3.8 \\
1.7\end{array}$ \\
\hline TC9 & $\left(+50^{\prime \prime},-90^{\prime \prime}\right)$ & NG & NG & NG & $\begin{array}{c}0.40 \\
16.5 \\
1.1\end{array}$ & $\begin{array}{c}3.5 \\
16.5 \\
1.2\end{array}$ & $\begin{array}{l}- \\
- \\
-\end{array}$ & $\begin{array}{l}- \\
- \\
-\end{array}$ \\
\hline TC10 & $\left(-20^{\prime \prime},-66^{\prime \prime}\right)$ & $\begin{array}{c}22.8 \\
0.4 \\
2.4\end{array}$ & $\begin{array}{c}18.9 \\
0.4 \\
2.8\end{array}$ & $\begin{array}{l}16.5 \\
0.8 \\
2.8\end{array}$ & $\begin{array}{l}2.0 \\
0.3 \\
0.8\end{array}$ & $\begin{array}{c}10.0 \\
0.3 \\
1.2\end{array}$ & $\begin{array}{l}2.5 \\
0.4 \\
1.4\end{array}$ & $\begin{array}{l}- \\
- \\
-\end{array}$ \\
\hline TC11 & $\left(-42^{\prime \prime},+30^{\prime \prime}\right)$ & $\begin{array}{c}16.1 \\
1.1 \\
2.0\end{array}$ & $\begin{array}{c}25.2 \\
1.1 \\
2.1\end{array}$ & $\begin{array}{c}16.2 \\
1.7 \\
2.6\end{array}$ & $\begin{array}{l}1.0 \\
1.2 \\
0.7\end{array}$ & $\begin{array}{l}2.9 \\
1.2 \\
1.4\end{array}$ & $\begin{array}{c}0.62 \\
1.1 \\
1.4\end{array}$ & $\begin{array}{l}- \\
- \\
-\end{array}$ \\
\hline TC13 & $\left(+240^{\prime \prime},+70^{\prime \prime}\right)$ & $\begin{array}{c}20.0 \\
4.2 \\
2.3\end{array}$ & $\begin{array}{c}23.0 \\
4.5 \\
2.9\end{array}$ & $\begin{array}{c}16.5 \\
4.7 \\
3.0\end{array}$ & $\begin{array}{c}<0.2 \\
- \\
-\end{array}$ & $\begin{array}{l}4.2 \\
3.8 \\
1.2\end{array}$ & $\begin{array}{l}1.3 \\
3.8 \\
1.2\end{array}$ & - \\
\hline
\end{tabular}

${ }^{1}$ L02. ${ }^{2}$ L00. ${ }^{3}$ Lefloch et al. (2008) (in prep.). $\dagger$ Corrected for beam dilution.

+ Measured on $\mathrm{SiO}$ as CS line profiles are self-absorbed.

Low resolution spectrum $\left(1 \mathrm{~km} \mathrm{~s}^{-1}\right)$; contamination from outflow wings and a second gas component on the line of sight. 


\section{Appendix A: Cores in the western filament}

\section{A.1. TC5}

TC5 is the brightest source discovered in our survey, with a peak flux of $\simeq 1 \mathrm{Jy} / 11^{\prime \prime}$ beam. It is located in the southernmost fragment $\left(\approx 810 M_{\odot}\right)$ of the western filament WF (Figs. $\left.1-\mathrm{A} .1\right)$. The isocontours of the $20 \mathrm{~cm}$ free-free emission show that the core lies at the edge of the W28 supernova remnant.

The core is elliptical with major and minor axis of $0.20 \mathrm{pc} \times$ $0.13 \mathrm{pc}$, respectively. The brightness peak is off-centered, suggesting the presence of multiple sources inside the continuum core. Indeed, SPITZER unveiled a cluster of sources in the dust central core (see right panel in Fig. A.1). The emission is strongly concentrated towards the peak. A mass of $100 M_{\odot}$ (about half the core mass) is contained within a $9^{\prime \prime}$ region, which indicates $\mathrm{H}_{2}$ densities $\left.n\left(\mathrm{H}_{2}\right) \simeq 8 \times 10^{6} \mathrm{~cm}^{-3}\right)$. The continuum source is not detected in the near-infrared with 2MASS. It however coincides with the IRAS pointsource 17589-2312. Comparison of the bolometric luminosity $\left(4000 L_{\odot}\right)$ to the millimeter luminosity yields a ratio $L_{\text {bol }} /\left(10^{3} \times L_{1.25}\right) \simeq 15$. This value is low and typical of those encountered in Class 0 protostellar cores. This testifies to the youth of the TC5 core.

The line profiles display broad wings tracing gas that reach velocity of $20 \mathrm{~km} \mathrm{~s}^{-1}$ with respect to the core, which testifies to the ongoing star formation at work in the core (Fig. A.2). The discovery of methanol maser emission confirms indeed that massive protostars or non-ionizing intermediate-mass protostars are forming in TC5 (Pestalozzi et al. 2005). Complementary interferometric observations are required to identify which of the continuum sources are powering the molecular outflows detected towards the core (Lefloch et al. 2008). The physical properties of TC5 are therefore very similar to those of TC 3 and TC4. At the high densities encountered in the central core of TC5, the typical gravitational free-fall time is $\tau=(3 \pi / 32 G \rho)^{1 / 2} \simeq 1.2 \times 10^{4} \mathrm{yr}$. The entire star-forming process taking place in the core is very recent.

\section{A.2. TC6}

The thermal dust emission shows a weakly contrasted condensation, without any dominant central condensation (Fig. A.3). Instead, there are several components barely resolved by the telescope beam, separated by typically $20^{\prime \prime}$. These subcondensations (TC6A-D) have low masses of typically 2-3 $M_{\odot}$.

Only a few molecular lines were observed towards TC6, at SEST and CSO. As the telescope main beam has a size comparable to the dimensions of the condensations, the mainbeam filling factor must be taken into account to accurately estimate the brightness of the CS lines and the density of the condensations. The filling factor $f$ is 0.81 and 0.65 for the $J=3 \rightarrow 2$ and the $J=2 \rightarrow 1$ transitions, respectively, assuming a Gaussian source distribution $f=\theta_{\mathrm{S}}^{2} /\left(\theta_{\mathrm{S}}^{2}+\theta_{\mathrm{B}}^{2}\right)$. Hence, the $\mathrm{CS}$ line brightness temperature of the $J=3 \rightarrow 2$ line components are $0.49 \mathrm{~K}$ and $0.35 \mathrm{~K}$, respectively, and $0.74 \mathrm{~K}$ and $0.65 \mathrm{~K}$ for the $J=2 \rightarrow 1$ line.

Neither ISO nor SPITZER detected pointlike sources towards TC6, which is consistent with TC6 being in a prestellar phase (see Fig. 1). Indeed, the mass of the subcondensations is much less than the virial equilibrium mass (see Sect. 7). Two kinematic components are detected in the CS lines (see Table 5). The line profiles are broader than those observed in the dust lanes of the nebula, and indicate a higher degree of turbulence.

\section{Appendix B: Cores in the eastern lane}

\section{B.1. TC8}

Figure 7 shows a second condensation gas in the eastern lane. Emission of the dense layers gas was observed in CS in the $J=2 \rightarrow 1$ transition at IRAM and in the $J=3 \rightarrow 2$ transition at SEST. Unfortunately, the fragment and TC8 could not be fully mapped.

The molecular emission peaks near the offset position $\left(+93.6^{\prime \prime},+88.6^{\prime \prime}\right)$ where a Class I protostar has been discovered by SPITZER (Rho et al. 2006). The lines of CO and its isotopologues have intensities comparable to those measured in TC1 (Fig. 10), indicating a kinetic temperature of about $30 \mathrm{~K}$. Due to incomplete mapping, we have estimated the physical conditions in the core from the emission at the position $\left(+90^{\prime \prime},+80^{\prime \prime}\right)$ close to the SPITZER continuum source. Because the core size is of the same order as the telescope beam size, the effects of the convolution with the main beam cannot be neglected, unlike the case of TC1, whose extent is typically twice the telescope beam size. The intensity of the CO $J=2 \rightarrow 1$ line is $\approx 0.75 \mathrm{~K}$ at the brightness peak. The beam filling factor reads $\theta_{\mathrm{s}}^{2} /\left(\theta_{\mathrm{s}}^{2}+\theta_{\mathrm{B}}^{2}\right)$ where $\theta_{\mathrm{s}}=20^{\prime \prime}$ and $\theta_{\mathrm{B}}=25.3^{\prime \prime}$ are the source and beam size (HPFW), respectively. A good estimate of the line brightness is obtained by dividing the main-beam brightness temperature by the beam filling factor. In the case of the CS $J=2 \rightarrow 1$ line, the beam filling factor is $\approx 0.385$, and the brightness temperature of the line is $1.9 \mathrm{~K}$. From the main-beam brightness temperature measured at SEST, we estimate a brightness temperature of $2.2 \mathrm{~K}$ for the $J=3 \rightarrow 2$ line (the beam size is $33.7^{\prime \prime}$ at this frequency). Hence, the line intensities compare well with those measured towards TC1.

As a conclusion, TC8 is characterized by molecular gas and dust emission which is less constrated than in TC1. However, the mass and the physical conditions in both condensations are rather similar. The dense gas region in TC8, as traced by CS, occupies a smaller part of the molecular gas fragment. The mass of the CS core represents only $25 \%$ of the fragment mass associated with TC8. In TC1, the CS core represents $80 \%$ of the whole mass. We interpret this difference as a result of a more effective photoionization and compression of TC1.

\section{B.2. TC13}

The eastern lane bends in the Southeastern direction beyond TC8 (see Fig. 1). This part of the dark lane contains lower amounts of gas and dust. As a consequence, it is detected in molecular gas emission ( $\mathrm{CO}$ isotopomers and CS) while it is missed by our $1.3 \mathrm{~mm}$ continuum mapping. A condensation of $55^{\prime \prime} \times 25^{\prime \prime}$ was discovered in this part of the filament (Fig. B.2). A Class 0/I source candidate (TC13) was identified by SPITZER, which lies in the outer layers of the condensation.

The CS $J=2 \rightarrow 1$ transition was detected at SEST. The line has a typical main-beam brightness intensity $T_{\mathrm{mb}}^{21}=0.6 \mathrm{~K}$. Once corrected for beam dilution (the beam size is comparable to the size of the core), we estimate a brightness temperature $T_{\mathrm{B}}^{21}=$ $1.3 \mathrm{~K}$ at the brightness peak (Fig. B.1). Assuming a standard relative abundance $[\mathrm{CS}] /\left[\mathrm{H}_{2}\right]=10^{-9}$, the $\mathrm{CS}$ column density is $N(\mathrm{CS}) \sim 6 \times 10^{12} \mathrm{~cm}^{-2}$. The gas density must be $\simeq 10^{5} \mathrm{~cm}^{-3}$ in order to account for the brightness of the CS $J=2 \rightarrow 1$ line. 


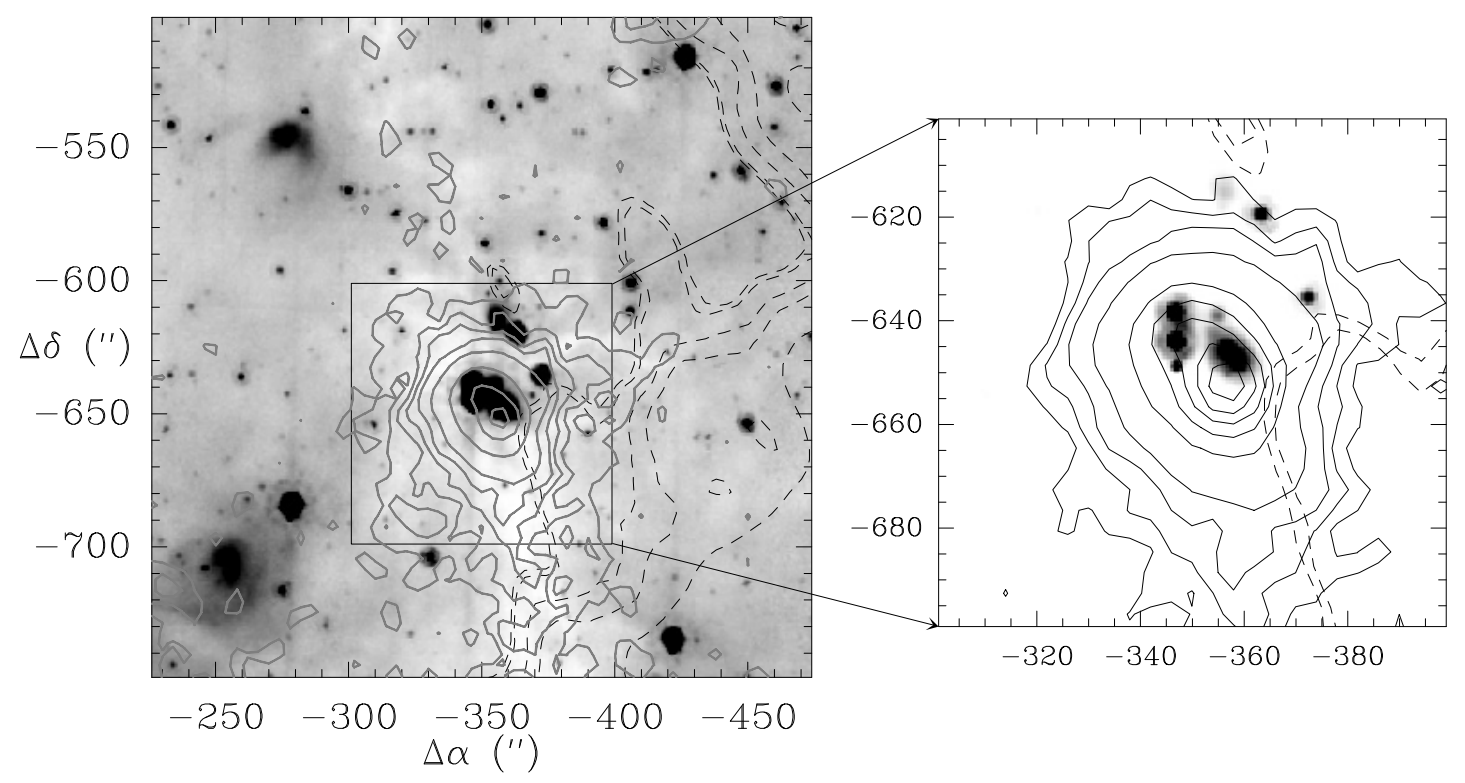

Fig. A.1. Contour map of thermal dust emission at $1.3 \mathrm{~mm}$ observed with the IRAM $30 \mathrm{~m}$ telescope superposed on an $8 \mu \mathrm{m}$ image of the region (greyscale). Contour levels are 25, 50, 75, 100, 150, 300, 450, 600, 750, $900 \mathrm{mJy} / 11^{\prime \prime}$ beam. The western filament WF is detected in absorption against the background. First contour and contour interval are $5 \mathrm{mJy} / 11^{\prime \prime}$ beam. A magnified view of the protostellar cluster associated with the TC5 core is presented in the right panel.

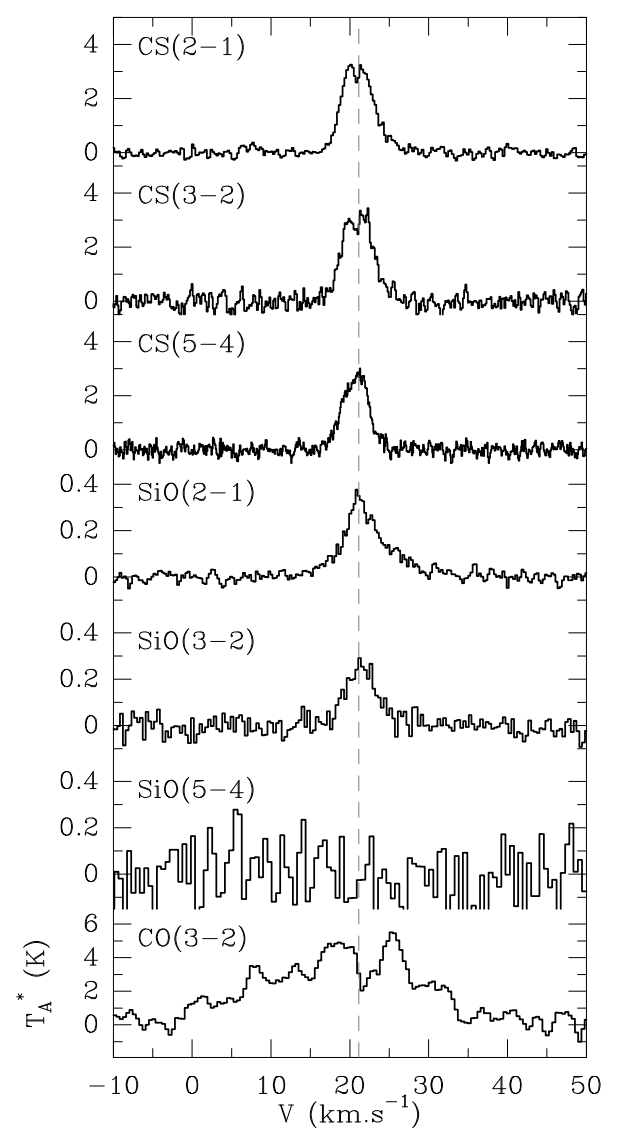

Fig. A.2. Molecular gas emission obtained towards TC5 in the millimeter transitions of $\mathrm{SiO}$ and $\mathrm{CS}$. Fluxes are expressed in units of antenna temperature.

\section{Appendix C: Cores in the southern region}

Two condensations, TC10 and TC11 respectively, could be identified in our molecular emission maps (see Fig. C.1). The two

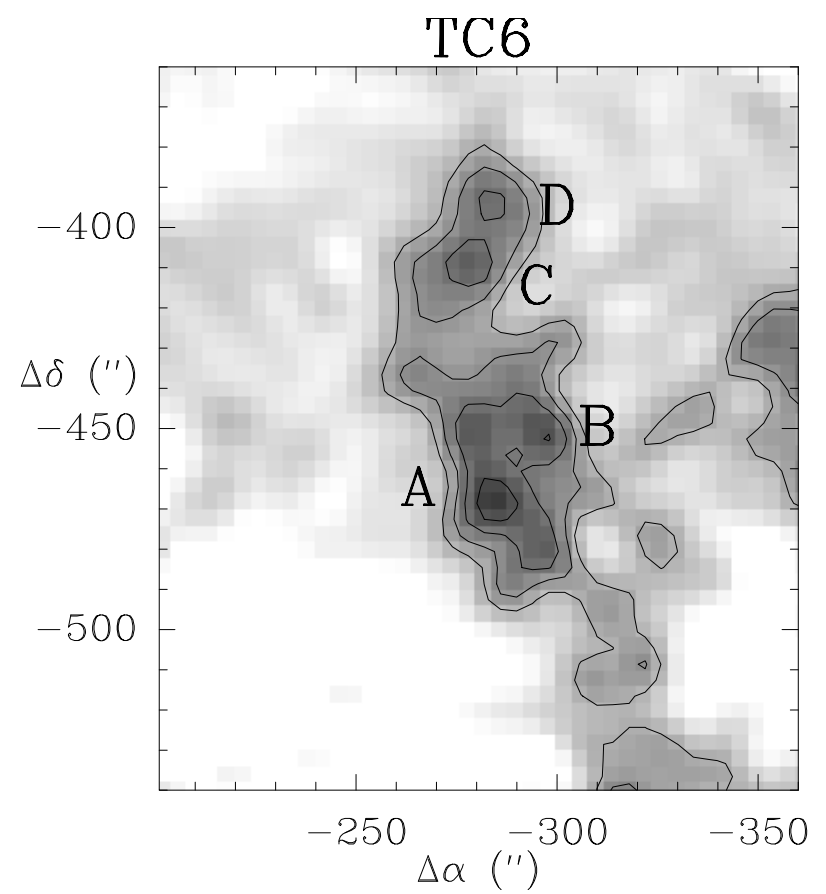

Fig. A.3. Thermal dust emission map of TC6 at $1.3 \mathrm{~mm}$ observed with the IRAM $30 \mathrm{~m}$ telescope. First contour and contour interval are $5 \mathrm{mJy} / 11^{\prime \prime}$ beam.

condensations are separated by $\approx 84^{\prime \prime}=0.7 \mathrm{pc}$. Only the first condensation is detected in the millimeter continuum. The overall emission is weak; the peak flux is about $25 \mathrm{mJy} / 11^{\prime \prime}$ beam, and the integrated flux is $25 \mathrm{mJy}$, implying a peak column density $N(\mathrm{H})=2.3 \times 10^{22} \mathrm{~cm}^{-2}$. The central core, defined by the contour at half power, has a typical size of $7^{\prime \prime}$ (beamdeconvolved) and a mass of $2.5 M_{\odot}$.

The molecular gas condensation, defined by the contour at half-power, has an elliptical shape with major (minor) axis of 
B. Lefloch et al.: Star formation in the Trifid Nebula, Online Material p 4

TC6

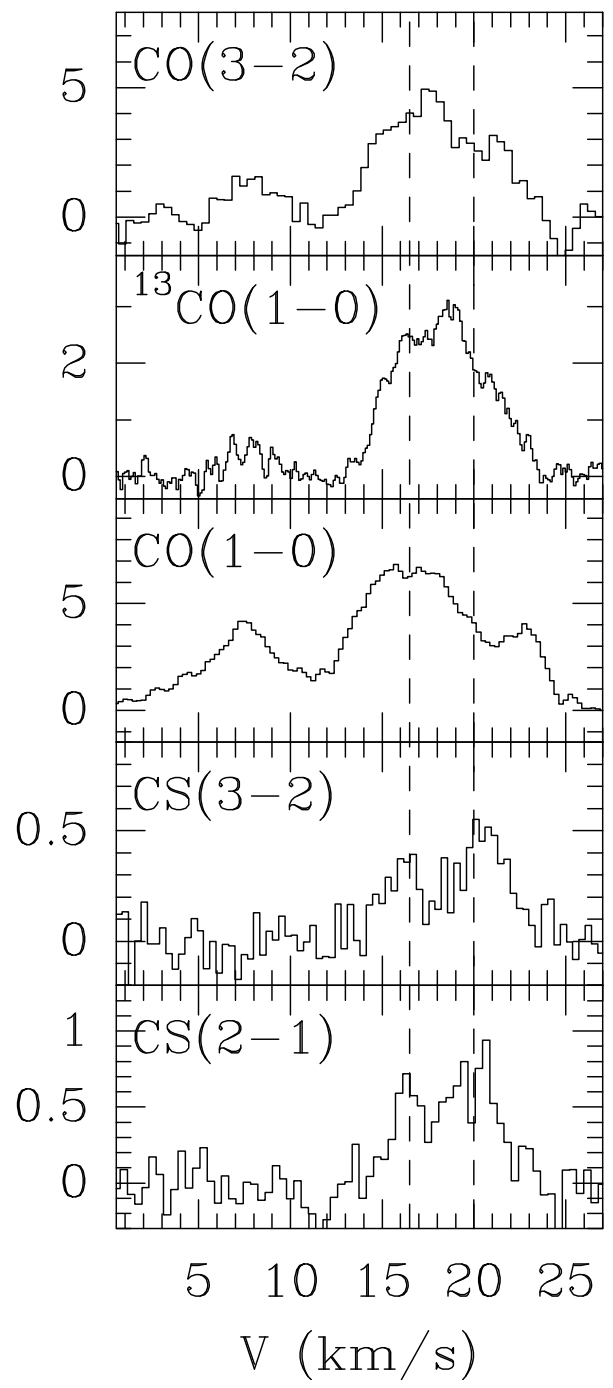

Fig. A.4. Millimeter line emission observed at SEST in TC6 at the offset position $\left(-280^{\prime \prime},-440^{\prime \prime}\right)$. The CS line intensities have been corrected for main beam dilution effects.

$80^{\prime \prime}\left(30^{\prime \prime}\right)$ contains a mass of $25 M_{\odot}$, almost one third of the material contained in the lane $\left(66 M_{\odot}\right)$.

Lines are somewhat narrower in the southern lane. We measure $T_{\mathrm{mb}}^{21}=2.5 \mathrm{~K}\left(\Delta v=1.4 \mathrm{~km} \mathrm{~s}^{-1}\right)$ (Fig. C.2). Assuming a standard relative abundance $[\mathrm{CS}] /\left[\mathrm{H}_{2}\right]=10^{-9}$, the CS line emission is consistent with $\mathrm{H}_{2}$ densities of $2.5 \times 10^{5} \mathrm{~cm}^{-3}$. In the Northern region, the molecular gas emission reveals TC11 as a larger condensation of larger dimensions and lower gas column density. Adopting the same physical conditions as in the South, we derive a peak column density $N\left(\mathrm{H}_{2}\right)=9 \times 10^{21} \mathrm{~cm}^{-2}$ and a mass of $6 M_{\odot}$ from the ${ }^{13} \mathrm{CO}$ data. No evidence of protostellar activity was detected in the southern lane. This is consistent with the mass of TC10 and TC11 being much lower than their virial mass.
TC13

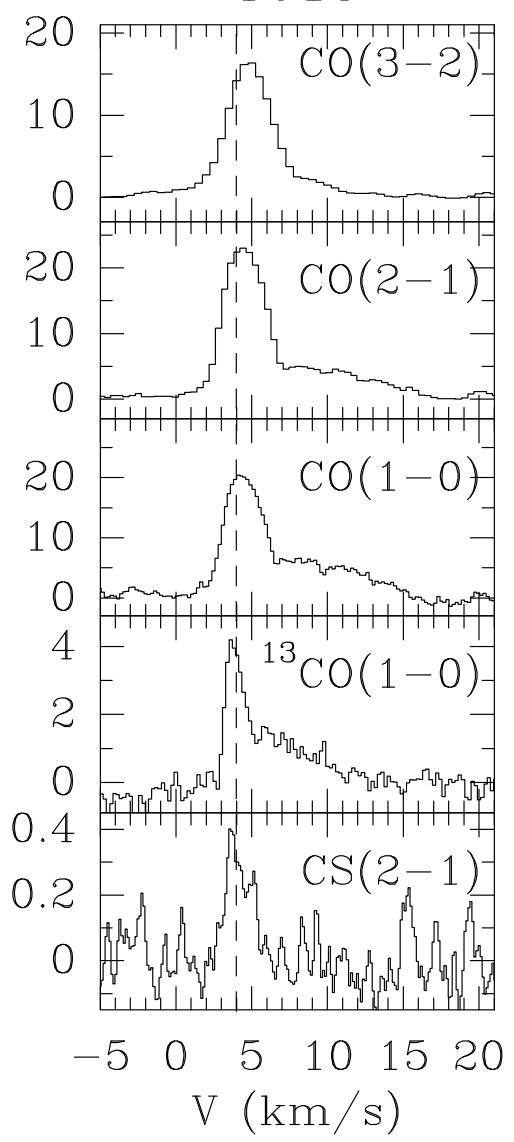

Fig. B.1. Molecular line spectra obtained at the emission peak of the molecular core TC13. Fluxes are expressed in units of antenna temperature. The dashed line marks the velocity of the main body gas.

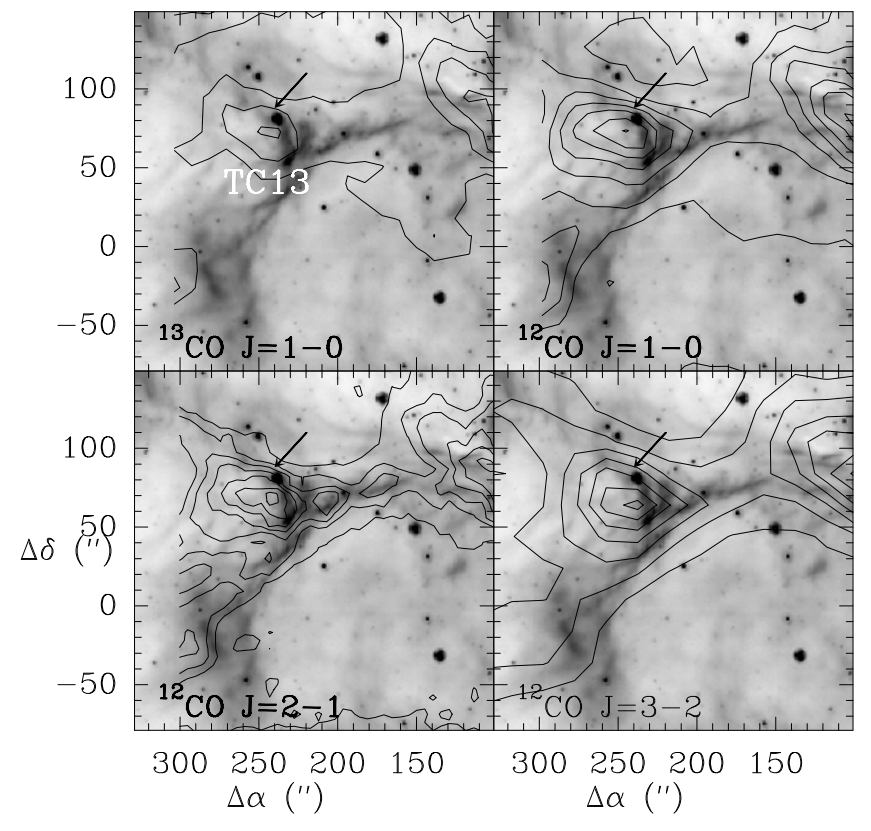

Fig. B.2. Molecular gas emission observed towards TC13, integrated between 2 and $8 \mathrm{~km} \mathrm{~s}^{-1}$. The arrow points to the position of the protostellar source. 
B. Lefloch et al.: Star formation in the Trifid Nebula, Online Material $p 5$

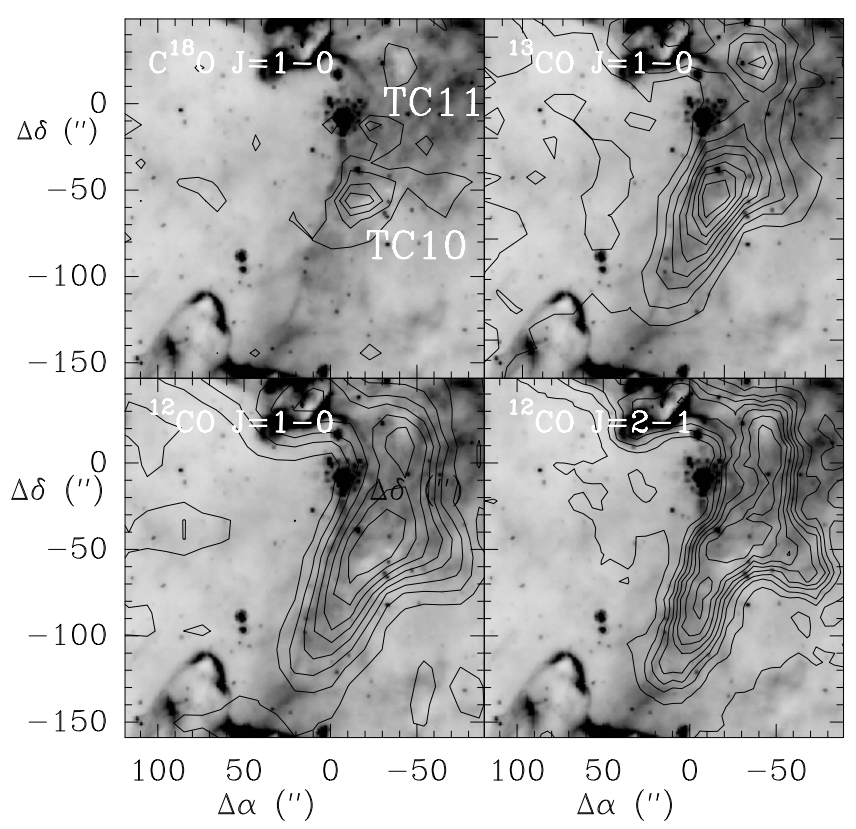

Fig. C.1. Molecular line emission in the southern lane region, integrated between -3 and $+3 \mathrm{~km} \mathrm{~s}^{-1}$, showing TC10 and TC11.

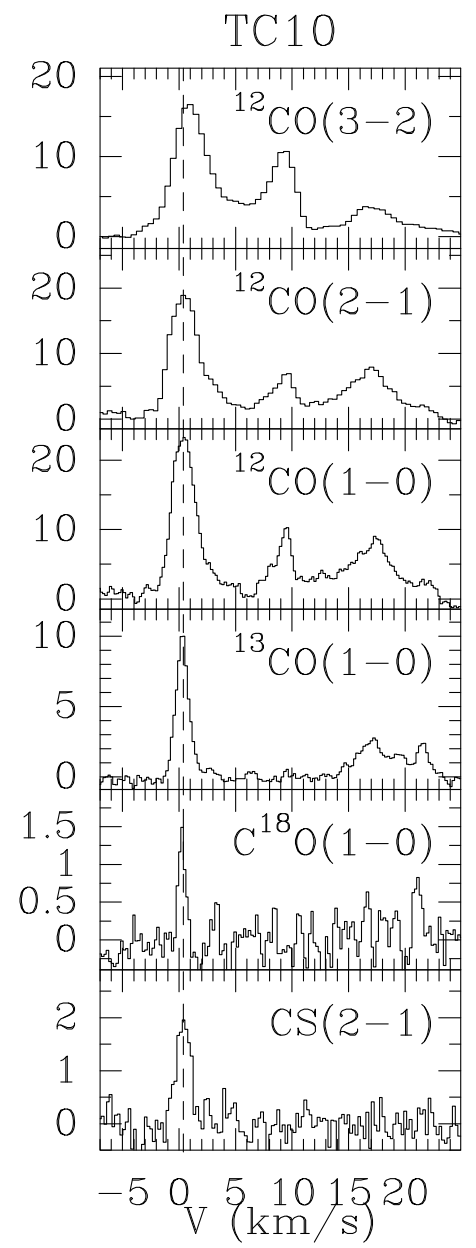

Fig. C.2. Molecular line spectra observed at the emission peak of TC10 in the southern lane. Fluxes are expressed in units of antenna temperature. 